University of Zurich

Department of Economics

Working Paper Series

ISSN 1664-7041 (print)

ISSN 1664-705X (online)

Working Paper No. 241

\title{
Exit Strategies for Monetary Policy
}

Aleksander Berentsen, Sébastien Kraenzlin and Benjamin Müller

First version: December 2016

This version: February 2018 


\title{
Exit Strategies for Monetary Policy*
}

\author{
Aleksander Berentsen ${ }^{\dagger}$ \\ University of Basel and Federal Reserve Bank of St. Louis \\ Sébastien Kraenzlin ${ }^{\ddagger}$ \\ Swiss National Bank \\ Benjamin Müller ${ }^{\S}$ \\ Swiss National Bank \\ Received Date; Received in Revised Form Date; Accepted Date
}

\begin{abstract}
In response to the financial crisis of $2007 / 08$, all major central banks decreased interest rates to historically low levels and created large excess reserves. Central bankers and academics currently discuss how to implement monetary policy, going forward. We find that paying interest on reserves (IOR) is optimal if the central bank has full fiscal support. If the central bank has no fiscal support, reducing reserves is optimal. This can be achieved by reserveabsorbing operations which hold the size of the balance sheet constant, or by selling assets which reduces the size of the balance sheet.
\end{abstract}

Keywords: Exit Strategies, Money Market, Repo, Monetary Policy, Interest Rates

JEL Classification: E40, E50, D83.

${ }^{*}$ The views expressed in this paper are those of the authors and do not necessarily represent those of the Swiss National Bank (SNB). We would like to thank the editor and an anonymous referee for their helpful comments and suggestions. Moreover, we are grateful for the comments received from participants at the workshop on Money, Banking and Payments at Fed St. Louis, the European System of Central Banks' Day-Ahead Conference, the Workshop on Market Liquidity at the University of Bern, the workshop on the New Normal for Monetary Policy Implementation at the Dutch National Bank, the SNB Brown Bag Seminar, the research seminar at the University of Nice, the research seminar at the Banque de France, and the research seminar at the University of California-Irvine. Finally, we would like to thank Morten Bech, Lucas Marc Fuhrer, Matthias Jüttner, Cyril Monnet, Guillaume Rocheteau, and Pedro Teles for their inputs. Berentsen thanks the SNB for research support. Most of this work was completed while affiliated with the SNB as an external advisor. The paper previously circulated under the title 'Exit Strategies and Trade Dynamics in Repo Markets'.

†aleksander.berentsen@unibas.ch; +41 6120712 05; University of Basel, Peter Merian-Weg 6, 4002 Basel, Switzerland.

¥sebastien.kraenzlin@snb.ch, Swiss National Bank, Boersenstrasse 15, P. O. Box, 8022 Zurich, Switzerland.

§benjamin.mueller@snb.ch, Swiss National Bank, Boersenstrasse 15, P. O. Box, 8022 Zurich, Switzerland. 


\section{Introduction}

Prior to the financial crisis of 2007/08, all major central banks created an environment in which the banking system was kept short of reserves, a so-called structural liquidity deficit. In such an environment, the central bank provides just enough reserves to ensure that financial intermediaries are able to meet their minimum reserve requirements. Consequently, reserves are scarce and the central bank can achieve the desired interest rate simply by changing the stock of reserves by a small amount via open market operations.

In response to the financial crisis of 2007/08 and the subsequent sovereign debt crisis, all major central banks lowered short-term interest rates to historically low levels and created large excess reserves via asset or foreign currency purchases. These quantitative easing (QE) measures led to a sharp increase of the central banks' balance sheets and moved the banking system from a structural liquidity deficit to a structural liquidity surplus environment. For that reason, central bankers and academics currently discuss how monetary policy should be implemented, going forward. The term 'exit strategies' refers to policies that allow central banks to control short-term interest rates in an environment with large excess reserves and three strategies are widely discussed: First, to leave excess reserves unchanged and pay interest on reserves (IOR). Second, to absorb reserves via instruments such as reverse repos, central bank bills, or term deposits. Third, to sell the assets purchased under QE. While the first strategy leaves the size of the central bank balance sheet and the quantity of reserves unchanged, the second strategy leaves the size of the balance sheet unchanged but reduces the quantity of reserves. The third strategy reduces the balance sheet and the quantity of reserves to pre-crisis levels.

Our goal with this paper is threefold: First, since central banks have little experience with these new policies, we construct a dynamic general equilibrium model with a secured money market and use it as a laboratory to study their properties. Second, to assess the quantitative effects of these policies on the economy, we calibrate the model to the Swiss franc repo market to study the welfare implications of these strategies. Furthermore, we evaluate them according to the following criteria: Their capacity to control the money market rate, their impact on the level of trading activity in the money market, their effect on inflation, and their tax implications. Third, since the future interaction between the fiscal and monetary authorities is uncertain, we assess the effects of these policies under two polar coordination regimes: 'Full fiscal support' and 'no fiscal support'.

Our main finding is that paying IOR is optimal if the central bank has full fiscal support. If the central bank has no fiscal support, reducing reserves is optimal. This reduction can be achieved by reserve-absorbing operations which hold the balance sheet constant, or by selling assets which reduces the balance sheet. It is important to note that under both regimes, optimality can be achieved with a larger central bank balance sheet than pre-crisis.

Further findings are as follows: First, all exit strategies allow central banks to raise interest rate to the targeted level. Second, the money market trading activity under IOR is almost inexistent, while trading activity re-emerges for all other policies and for both regimes. Third, none of our experiments require a negative real transfer (a tax) to satisfy the consolidated government budget constraint. Nevertheless, IOR, term deposits, central bank bills and reverse repos involve substantial interest payments from the central bank to financial intermediaries. This is not the case if assets are sold. These additional findings do not affect our welfare measure, but they may be relevant if the central bank values criteria such as money market activity in view of the current benchmark interest rate reform efforts or if political economy factors such as making large interest payments to (a few) financial intermediaries are taken into account.

The findings of this paper contribute to the current discussion on the new regime for monetary policy implementation. In particular, it provides guidance whether monetary policy should be implemented in an excess reserve environment with IOR going forward or whether central banks should return to the pre-crisis monetary policy implementation by absorbing reserves and/or reducing the balance sheet size. For example, the Federal Reserve is currently operating a combination of IOR and reverse repos to control the federal funds effective rate. Moreover, it has announced to reduce its balance sheet size. ${ }^{1}$ The Swiss National Bank (SNB) used central bank bills and reverse repos in 2010 and 2011 to absorb reserves.

Literature. Our paper is related to a set of papers studying the trade dynamics in the markets for reserves with an attempt to simulate economies with large excess reserves and the central bank aiming to raise interest rates. Afonso and Lagos (2015) develop a model of the federal funds market - an unsecured money market for central bank reserves. In their modeling approach, they explicitly take into account the search and bargaining frictions that are key characteristics of this market. They calibrate their model and evaluate the effectiveness of IOR in controlling the federal funds rate. Armenter and Lester (2016) provide a model that captures the institutional details of the US money market that are

\footnotetext{
${ }^{1}$ See, for instance, the Federal Reserve's "Policy Normalization Principles and Plans" (FOMC, September 2014), the considerations on the Federal Reserve's "Long-Run Monetary Policy Implementation Framework" (FOMC, November 2016), and the plan for the Federal Reserve's balance sheet reduction process (FOMC, 2017a and FOMC, 2017b). Reasons for this particular combination of IOR and reverse repos are the Federal Reserve's access policy and the structure of the US money market.
} 
relevant for the Federal Reserve's ability to raise the federal funds rate in an environment with large excess reserves. They also calibrate the model and run monetary policy experiments to understand the market reactions when the Federal Reserve hikes rates in a controlled environment. Related literature on general equilibrium interbank models include Bech and Monnet (2016) and Berentsen and Monnet (2008).

Our paper is also related to Rocheteau et al. (2017), who provide a theoretical model of open market operations (OMOs). As in our paper, different types of central bank interventions and their effects are investigated. Rocheteau et al. (2017) argue that OMOs involve the exchange of two financial assets, cash (in our paper central bank reserves) and bonds. Since both are used as a medium of exchange or as collateral that facilitates trading, they convey liquidity. Due to differences in acceptability and pledgeability arising from informational frictions, the authors endogenize the liquidity characteristics of cash and bonds and show that the effect of OMO crucially depends on their relative liquidity. In that regard, the authors show that there are instances where OMOs may be the wrong remedy to address a liquidity shortage.

Finally, our paper is related to the literature studying optimal monetary policy and the implications of excess reserves for inflation. Ennis (2017), Williamson (2016), and Reis (2016) study environments where banks hold large excess reserves and the central bank pays IOR. In such an environment, combined with capital constraint banks, Ennis (2017) finds that a price level indeterminacy may arise. That is, large excess reserves may not need to be associated with a higher price level. Williamson (2016) investigates two policies, one in which the central bank pays IOR and one where the level of reserves is reduced in a two-sector banking model. While the effect on nominal interest rates is similar, the effect on welfare can be different with the key driver being balance sheet costs imposed on banks. Policies that reduce banks' balance sheet costs are superior from a welfare perspective than paying IOR. Examples are central bank balance sheet reductions (e.g. unwinding of $\mathrm{QE}$ ) or reserve-absorbing facilities accessible for non-banks (see, for instance, the Federal Reserves's overnight reverse repo facility). Reis (2016) studies the link between the level of outstanding reserves and inflation. The paper argues that in an environment where banks are saturated with reserves, only the IOR and not the size of the balance sheet has an effect on inflation. Thus, the paper suggests that central banks can use IOR to control inflation and use QE for other monetary policy goals such as financial stability. Our paper adds to this literature because we show that these findings crucially depend on the interaction between fiscal and monetary policy.

This paper is organized as follows: Section 2 develops the theory and Section 3 presents the quantitative analysis. Section 4 analyses exit strategies. Section 5 presents a comparison of all exit strategies when the central bank implements an interest rate target and Section 6 concludes. The Web Appendix includes the proofs, discusses the consolidated government budget constraint and welfare, describes the data, the calibration, as well as the simulation, discusses comparative statics, and documents the institutional details of the Swiss franc repo market.

\section{Theory}

The theoretical model is a dynamic general equilibrium model with a secured money market developed in Berentsen et al. (2014a and 2014b). It is adapted to take account of the elementary features of the Swiss franc repo market, but many other money markets have similar characteristics which are the following: ${ }^{2}$ First, at the beginning of the day all outstanding overnight loans are repaid. Second, the Swiss franc repo market operates between 7 am and 6 pm. Third, the SNB controls the stock of reserves by conducting open market operations, typically at 9 am. Fourth, after the money market has closed, the SNB operates its lending facility (discount window) for an additional 15 minutes. This is the last opportunity for financial intermediaries (FIs) to acquire overnight reserves for the same business day to settle outstanding short positions in the payment system.

In contrast to a growing body of literature, which models money markets as over-the-counter (OTC) markets that are characterized by search and bargaining frictions, we model the money market as a competitive market. We opted for this modelling strategy after carefully inspecting the institutional details of trading in the Swiss franc repo market which is characterized by very few informational frictions and close to zero counterparty risk. The study and findings also apply to other currency areas, since there is a trend towards shifting money market trading onto transparent (centrally-cleared) electronic trading platforms that reduce informational frictions (see ICMA, 2014).

\footnotetext{
${ }^{2}$ The Swiss franc repo market is the secured money market for central bank reserves. We model this market because this allows us to benefit from outstanding data quality, featuring detailed information on more than 100,000 overnight transactions. In contrast to many other studies, there is no need to identify transactions from payment system data applying the Furfine (2000) algorithm which has known caveats (see Armantier and Copeland, 2012). A detailed description of the Swiss franc repo market is provided in the Web Appendix. The institutional details of the euro area money market and monetary policy implementation by the European Central Bank share the same elements (see Berentsen and Monnet, 2008). For the US, some instutional details differ mainly because it is a segmented market. See Armenter and Lester (2016) for an overview.
} 


\subsection{Environment}

To reproduce the above sequence it is assumed that in each period three perfectly competitive markets open sequentially (see Figure 1): A settlement market, where credit contracts are settled and a generic good is produced and consumed; a money market, where borrowing and lending of reserves on a secured basis take place; and a goods market, where production and consumption of a specialized good take place. All goods are perfectly divisible and nonstorable, which means that they cannot be carried from one market to the next.

Figure 1: Sequence of markets ${ }^{\mathrm{a}}$

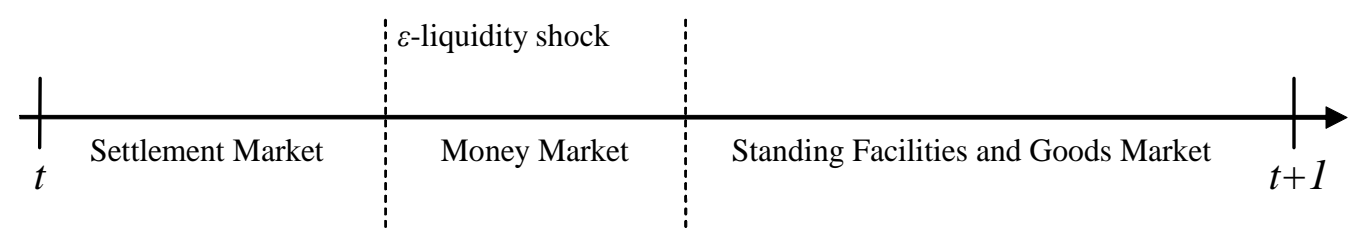

${ }^{a}$ In each period, three perfectly competitive markets open sequentially: a settlement market, a money market, and

a goods market. At the beginning of the money market, an $\varepsilon$-liquidity shock realizes.

There are two types of agents: firms and FIs. Both agent types are infinitely-lived and each of them has the measure 1. The focus of our attention will be on the FIs, since firms play a subordinate role in the model. They are only needed to obtain a first-order condition in the goods market.

Time is discrete and the discount factor across periods for both agent types is $\beta=(1+r)^{-1}<1$, where $r$ is the time rate of discount. There are two perfectly divisible financial assets: reserves and one-period, nominal discount bonds. One bond pays off one unit of reserves in the settlement market of the following period. Bonds are default-free and book-keeping entries; i.e., no physical object exists.

The three markets are now discussed backwards. In the goods market, the specialized good is produced by firms and consumed by FIs. ${ }^{3}$ Firms incur a utility cost $c\left(q_{s}\right)=q_{s}$ from producing $q_{s}$ units and FIs gain utility $\varepsilon u(q)$ from consuming $q$ units, where $u(q)=\log (q)$, and $\varepsilon$ is a preference shock that affects the liquidity needs of FIs. The preference shock has a continuous distribution $F(\varepsilon)$ with support $(0, \infty]$, is i.i.d. across FIs, and is serially uncorrelated.

To introduce a microfoundation for the demand for reserves, it is assumed that reserves are the only medium of exchange in the goods market. This is motivated by the assumption that FIs are anonymous in the goods market and that none of them can commit to honor intertemporal promises. ${ }^{4}$ Since bonds are intangible objects, only reserves can be used as a medium of exchange in the goods market. Furthermore, claims to bonds cannot be used as a medium of exchange, since agents can perfectly and costlessly counterfeit such claims by assumption (see Lester et al., 2012). In other words, bonds are illiquid in the goods market.

At the beginning of the money market, FIs hold a portfolio of reserves and bonds and then learn the current realization of the liquidity shock. Based on this information, they adjust their reserves holdings by either trading in the money market or at the standing facilities. The central bank is assumed to have a record-keeping technology that keeps track of all trades in the money market.

In the settlement market, a generic good is produced and consumed by firms and FIs. Firms and FIs have a constantreturns-to-scale production technology, where one unit of the good is produced with one unit of labor generating one unit of disutility. Thus, producing $h$ units of goods implies disutility $-h$. Furthermore, the utility of consuming $x$ units of goods is assumed to yield utility $x$. As in Lagos and Wright (2005), these assumptions yield a degenerate distribution of portfolios at the beginning of the money market.

\subsection{Central bank fiscal support}

It is assumed that there is a fiscal authority that has the power to levy a lump-sum tax, with $T$ denoting the nominal lumpsum transfer to each FI in period $t$ ( $T<0$ is a lump-sum tax). The government issues one-period bonds $B_{G}=\bar{B}_{g}+B_{g}$ that are either held by the central bank, $\bar{B}_{g}$, or the FIs, $B_{g}$. The government budget constraint at time $t$ satisfies

$$
\phi T=\phi \rho B_{G}^{+}-\phi B_{G}+\phi T_{c},
$$

\footnotetext{
${ }^{3}$ In practice, households consume and hold money on accounts at FIs. The $\varepsilon$-shock can be interpreted as a liquidity shock for FIs which originates from preference or technology shocks experienced by their customers. In order to simplify the model, we abstract from this additional layer, by assuming that our FIs are endowed with the same preferences as potential households.

${ }^{4}$ In practice, households and firms operate in the goods market and the demand for reserves arises because they are anonymous to each other (see also Footnote 3).
} 
where $\phi$ is the price of reserves in terms of the generic good that is traded in the settlement market and $T_{c}$ is a nominal transfer from the central bank to the government representing dividend or remittance payments from the monetary authority to the fiscal authority. Note that $T_{c}$ can be negative. The price of newly issued bonds is $\rho=1 /(1+i)$, where $i$ denotes the nominal interest rate. Note that throughout the paper, the plus sign is used to denote the next-period variables.

In the settlement market, the central bank controls the stock of reserves and issues one-period bonds (central bank bills) denoted $B_{c}$. Central bank bills can be used to absorb reserves and thus serve as an exit instrument. Government bonds and central bank bills are both riskless and promise to pay one unit of reserves in the following period. Consequently, they are priced equally, namely, $\rho=1 /(1+i)$.

In the goods market, the central bank operates two standing facilities. First, it offers a lending facility, where the central bank offers nominal loans $\ell$ at an interest rate $i_{\ell}$. Second, the central bank offers a deposit facility, where it pays interest rate $i_{d}$ on nominal deposits $d$ with $i_{\ell} \geq i_{d}$. An FI that borrows $\ell$ units of reserves at the lending facility in the goods market in period $t$ repays $\left(1+i_{\ell}\right) \ell$ units of reserves in the settlement market of the following period. Also, an FI that deposits $d$ units of reserves at the deposit facility in the goods market of period $t$ receives $\left(1+i_{d}\right) d$ units of reserves in the settlement market of the following period. Finally, the central bank operates at zero cost. ${ }^{5}$

The central bank's budget constraint satisfies

$$
\phi T_{c}=\phi M^{+}-\phi M+\left(\rho \phi B_{c}^{+}-\phi B_{c}\right)-\left(\phi \rho \bar{B}_{g}^{+}-\phi \bar{B}_{g}\right)-\left(1 / \rho_{d}-1\right) \phi D+\left(1 / \rho_{\ell}-1\right) \phi L,
$$

where $M$ is the stock of reserves at the beginning of the current-period settlement market. The quantity $B_{c}$ is the stock of central bank bills at the beginning of the current-period settlement market and $B_{c}^{+}$is the stock of bills at the beginning of the next-period settlement market. Since in the settlement market total loans, $L$, are repaid and total deposits, $D$, are redeemed, the difference $\left(1 / \rho_{\ell}-1\right) L-\left(1 / \rho_{d}-1\right) D$ is the central bank's revenue from operating the standing facilities.

Throughout the paper, we distinguish between 'full fiscal support' and 'no fiscal support'. With full fiscal support, we assume that the central bank chooses its policy independent of fiscal policy considerations and thus neglects the impact of its policy on $T_{c}$. With no fiscal support, the central bank chooses its policy, taking $T_{c}$ as given. In most monetary models, $T_{c}$ and through (1) also $T$ passively adjust to satisfy the government and the central bank budget constraints. As discussed in Hall and Reis (2015) and further elaborated in Del Negro and Sims (2015), this effectively means that fiscal policy fully supports central bank actions and the central bank is fully independent of fiscal policy considerations. As shown in these papers and also in our analysis below, neglecting the interaction between fiscal and monetary policy has important implications. ${ }^{6}$

In the context of our model, full fiscal support means that the monetary authority can independently choose the interest rates at the standing facilities, $\rho_{d}$ and $\rho_{\ell}$, and the inflation target since the implications for fiscal policy can be neglected. From (2), any combination of these monetary policy instruments determines the real transfer $\phi T_{c}$. Then, given $\phi T_{c}$, the fiscal authority needs to adjust its spending or its tax revenues $\phi T$ to satisfy (1). ${ }^{7}$ No fiscal support is modelled such that the central bank takes $\phi T_{c}$ as given; i.e., (2) is a constraint.

By combining (1) and (2), the following consolidated government budget constraint is obtained:

$$
\phi T=\phi M^{+}-\phi M+\phi \rho B^{+}-\phi B-\left(1 / \rho_{d}-1\right) \phi D+\left(1 / \rho_{\ell}-1\right) \phi L,
$$

where $B=B_{g}+B_{c}$ is the aggregate quantity of bonds held by FIs.

Note that $T$ is assumed to be a lump-sum transfer. This assumption is an appropriate approximation because $T_{c}$ is small in reality and in particular, it is small relative to the total government tax income. Accordingly, effects from distortionary taxations that arise from $T_{c}$ are small and hence we ignore it.

\subsection{Welfare}

In the Web Appendix, it is shown that in a steady state the expected lifetime utility $W$ of an FI satisfies

$$
(1-\beta) W=\int\left[\varepsilon u\left(q_{\varepsilon}\right)-q_{\varepsilon}\right] d F(\varepsilon) .
$$

\footnotetext{
${ }^{5}$ There is a difference in timing between deposit to and lending from the standing facilities. FIs borrow from the lending facility before they trade with firms. Firms and FIs deposit reserves at the deposit facility after the trade.

${ }^{6}$ For related but alternative studies of the interaction between fiscal and monetary policy, see Sargent and Wallace (1981), Leeper and Leith (2016) and Reis (2017).

${ }^{7}$ This is the current practice of the Federal Reserve. Under the Federal Reserve's policy, the earnings of each Federal Reserve Bank are distributed to the US Treasury, after having taken into account the costs of operations, payment of dividends, and other items.
} 
It is routine to show that the first-best consumption quantities in the goods market satisfy $q_{\varepsilon}^{*}=\varepsilon$ for all $\varepsilon$. Note that $W$ only depends on the consumption quantities in the goods market. Utilities from consuming and producing goods in the settlement market always add up to zero.

\subsection{Agents' decisions}

In this section, the decision problems of FIs and firms are studied. For this purpose, we let $P$ denote the price of goods in the settlement market and define $\phi \equiv 1 / P$. Furthermore, $p$ denotes the price of goods in the goods market.

Settlement market. $V_{S}(m, b, \ell, d, z)$ denotes the expected value of entering the settlement market with $m$ units of reserves, $b$ bonds, $\ell$ loans from the lending facility, $d$ deposits from the deposit facility, and $z$ loans from the money market. Note that $b=b_{g}+b_{c}$, where $b_{g}$ and $b_{c}$ are, respectively, the government bonds and central bank bills held by an FI. Since both types of bonds redeem a unit of reserves in the following settlement market, and both can be used as collateral and are riskless, they trade at par. Since FIs are indifferent between the two, they are assumed to hold them all in the same proportion.

$V_{M}(m, b)$ denotes the expected value from entering the money market with $m$ units of reserves and $b$ units of collateral prior to the realization of the liquidity shock $\varepsilon$. For notational simplicity, the dependence of the value function on the time index $t$ is suppressed. In the settlement market, the problem of an agent is

$$
\begin{gathered}
V_{S}(m, b, \ell, d, z)=\max _{h, x, m \prime, b \prime} x-h+V_{M}\left(m^{\prime}, b^{\prime}\right) \\
\text { s.t. } x+\phi m^{\prime}+\phi \rho b^{\prime}=h+\phi m+\phi b+\phi d / \rho_{d}-\phi \ell / \rho_{\ell}-\phi z / \rho_{m}+\phi \tau,
\end{gathered}
$$

where $h$ is hours worked in the settlement market, $x$ is consumption of the generic good, $m^{\prime}\left(b^{\prime}\right)$ is the amount of reserves (bonds) brought into the money market, and $\tau$ is a lump-sum transfer. Using the budget constraint to eliminate $x-h$ in the objective function, one obtains the first-order conditions

$$
\begin{aligned}
V_{M}^{m^{\prime}} & \leq \phi\left(=\text { if } m^{\prime}>0\right) \\
V_{M}^{b^{\prime}} & \leq \phi \rho\left(=\text { if } b^{\prime}>0\right) .
\end{aligned}
$$

$V_{M}^{m^{\prime}} \equiv \frac{\partial V_{M}\left(m^{\prime}, b^{\prime}\right)}{\partial m^{\prime}}$ is the marginal value of taking an additional unit of reserves into the money market. Since the marginal disutility of working is one, $-\phi$ is the utility cost of acquiring one unit of reserves in the settlement market. $V_{M}^{b^{\prime}} \equiv \frac{\partial V_{M}\left(m^{\prime}, b^{\prime}\right)}{\partial b^{\prime}}$ is the marginal value of taking additional bonds into the money market. The term $-\phi \rho$ is the utility cost of acquiring one unit of bonds in the settlement market. The implication of (5) and (6) is that all FIs enter the money market with the same amount of reserves and the same quantity of bonds. The same is true for firms, since in equilibrium they will bring no reserves into the money market.

The envelope conditions are

$$
V_{S}^{m}=V_{S}^{b}=\phi ; V_{S}^{d}=\phi / \rho_{d} ; V_{S}^{\ell}=-\phi / \rho_{\ell} ; V_{S}^{z}=-\phi / \rho_{m},
$$

where $V_{S}^{j}$ is the partial derivative of $V_{S}(m, b, \ell, d, z)$ with respect to $j=m, b, \ell, d, z$.

Money and goods markets. The money market is perfectly competitive so that the money market interest rate $i_{m}$ clears the market. Let $\rho_{m} \equiv 1 /\left(1+i_{m}\right)$. All transactions are restricted to overnight transactions. An FI that borrows one unit of reserves in the money market repays $1 / \rho_{m}$ units of reserves in the settlement market of the following period. Also, an FI that lends one unit of reserves receives $1 / \rho_{m}$ units of reserves in the settlement market of the following period.

Firms produce goods in the goods market with linear cost $c(q)=q$ and consume in the settlement market, obtaining linear utility $U(x)=x$. It is straightforward to show that they are indifferent as to how much they sell in the goods market if

$$
p \beta \phi^{+} / \rho_{d}=1,
$$

where $\phi^{+}$is the price of reserves in the next-period settlement market. Since the focus is on a symmetric equilibrium, it is assumed that all firms produce the same amount. With regard to bond holdings, it is straightforward to show that, in equilibrium, firms are indifferent to holding any bonds if the Fisher equation holds, and that they will hold no bonds if the yield on bonds does not compensate them for inflation or time discounting. Thus, for brevity of analysis, it is 
assumed that firms carry no bonds across periods. Note that firms are allowed to deposit their proceeds from sales at the deposit facility which explains the deposit factor $\rho_{d}$ in (8). ${ }^{8}$ Furthermore, it is also clear that they will never acquire reserves in the settlement market, so that for them, $m^{\prime}=0$.

An FI can borrow or lend at the money market rate $i_{m}$ or use the standing facilities. For an FI with preference shock $\varepsilon$, which enters the money market with $m$ units of reserves and $b$ units of bonds, the indirect utility function $V_{M}(m, b \mid \varepsilon)$ satisfies

$$
\begin{array}{cc} 
& V_{M}(m, b \mid \varepsilon)=\max _{q_{\varepsilon}, z_{\varepsilon}, d_{\varepsilon}, \ell_{\varepsilon}} \varepsilon u\left(q_{\varepsilon}\right)+\beta V_{S}\left(m+\ell_{\varepsilon}+z_{\varepsilon}-p q_{\varepsilon}-d_{\varepsilon}, b, \ell_{\varepsilon}, d_{\varepsilon}, z_{\varepsilon}\right) \\
\text { s.t. } \quad & m+z_{\varepsilon}+\ell_{\varepsilon}-p q_{\varepsilon}-d_{\varepsilon} \geq 0, \rho_{m} \theta b-z_{\varepsilon} \geq 0, \rho_{m} \theta b-z_{\varepsilon}-\left(\rho_{m} / \rho_{\ell}\right) \ell_{\varepsilon} \geq 0, d_{\varepsilon} \geq 0 .
\end{array}
$$

The first inequality is the FI's budget constraint in the goods market. The second inequality is the collateral constraint in the money market, and the third inequality is the collateral constraint at the lending facility where $\theta$ denotes the fraction of bonds that FIs use as collateral in the money market. We introduce $\theta$ here, because it is relevant for the calibration. ${ }^{9}$ The last inequality reflects the fact that deposits cannot be negative. Let $\beta \phi^{+} \lambda_{\varepsilon}$ denote the Lagrange multiplier for the first inequality, $\beta \phi^{+} \lambda_{z}$ denote the Lagrange multiplier for the second inequality, $\beta \phi^{+} \lambda_{\ell}$ denote the Lagrange multiplier for the third inequality, and $\beta \phi^{+} \lambda_{d}$ denote the Lagrange multiplier for the last inequality.

FIs use the standing facilities if, and only if, $\rho_{\ell}=\rho_{m}$ or $\rho_{d}=\rho_{m}$. For brevity of the analysis, in the characterization below, these two cases are ignored by assuming $\rho_{d}>\rho_{m}>\rho_{\ell}$. In this case, $d_{\varepsilon}=0$ and $\ell_{\varepsilon}=0$. Furthermore, the second and third inequalities are equal and so the third inequality can be ignored without loss in generality.

Using (7), the first-order condition for $z_{\varepsilon}$ is

$$
1+\lambda_{\varepsilon}=\lambda_{z}+\frac{1}{\rho_{m}}
$$

If $\rho_{d}>\rho_{m}>\rho_{\ell},(7)$ and (8) can be used to write the first-order conditions for $q_{\varepsilon}$ as follows:

$$
\varepsilon u^{\prime}\left(q_{\varepsilon}\right)-\rho_{d} / \rho_{m}=\rho_{d} \lambda_{z} .
$$

Lemma 1 characterizes the optimal borrowing and lending decisions and the quantity of goods obtained by an $\varepsilon-\mathrm{FI}$ :

Lemma 1 There exist critical values $\varepsilon_{1}$, $\varepsilon_{2}$, with $0 \leq \varepsilon_{1} \leq \varepsilon_{2}$, such that the following is true: if $0 \leq \varepsilon \leq \varepsilon_{1}$, an FI lends reserves in the money market; if $\varepsilon_{1} \leq \varepsilon \leq \varepsilon_{2}$, an FI borrows reserves and the collateral constraint is nonbinding; if $\varepsilon_{2} \leq \varepsilon$, an FI borrows reserves and the collateral constraint is binding. The critical values in the money market solve

$$
\varepsilon_{1}=\frac{\rho_{d}}{\rho_{m}} \frac{m}{p}, \text { and } \varepsilon_{2}=\varepsilon_{1}\left(1+\rho_{m} \frac{\theta b}{m}\right) .
$$

Furthermore, the amount of borrowing and lending by an FI with a liquidity shock $\varepsilon$ and the amount of goods purchased by the FI satisfy:

$$
\begin{array}{lll}
q_{\varepsilon}=\varepsilon \rho_{m} / \rho_{d}, & z_{\varepsilon}=p\left(\rho_{m} / \rho_{d}\right)\left(\varepsilon-\varepsilon_{1}\right), & \text { if } 0 \leq \varepsilon \leq \varepsilon_{1} \\
q_{\varepsilon}=\varepsilon \rho_{m} / \rho_{d}, & z_{\varepsilon}=p\left(\rho_{m} / \rho_{d}\right)\left(\varepsilon-\varepsilon_{1}\right), & \text { if } \varepsilon_{1} \leq \varepsilon \leq \varepsilon_{2}, \\
q_{\varepsilon}=\varepsilon_{2} \rho_{m} / \rho_{d}, & z_{\varepsilon}=\rho_{m} \theta b, & \text { if } \varepsilon_{2} \leq \varepsilon .
\end{array}
$$

Proof of Lemma 1. See Web Appendix.

Consumption quantities by FIs are increasing in $\varepsilon$ in the interval $\varepsilon \in\left[0, \varepsilon_{2}\right)$ and are flat for $\varepsilon \geq \varepsilon_{2}$. Note that $\rho_{m} / \rho_{d} \leq 1$, which means that the quantities consumed by FIs are always below the first-best quantities, unless $\rho_{m}=\rho_{d}$. FIs with a low liquidity shock $\varepsilon$ are lenders. Furthermore, there are two types of borrowers. FIs with an intermediate liquidity shock borrow small amounts of reserves so that the collateral constraint is nonbinding. FIs with a high liquidity shock would like to borrow large amounts of reserves, but their collateral constraint is binding.

\subsection{Equilibrium}

We focus on symmetric stationary equilibria with strictly positive demand for nominal bonds and reserves. Such equilibria meet the following requirements: (i) FIs' and firms' decisions are optimal, given prices; (ii) The decisions are

\footnotetext{
${ }^{8}$ This assumption reflects the fact that, in practice, firms hold cash from the proceeds of sales on their deposit account at FIs. FIs, in turn, hold these deposits on their reserve account at the central bank.

${ }^{9}$ In practice, only a fraction of the FIs' bonds holdings are used as collateral in the money market. In the case of the Swiss franc repo market, these bonds are earmarked in the securities' account held at the triparty-agent (see Web Appendix).
} 
symmetric across all firms and symmetric across all FIs with the same preference shock; (iii) All markets clear; (iv) All real quantities are constant across time; (v) The law of motion for the stock of reserves (2) holds in each period.

Let $\gamma \equiv M^{+} / M$ denote the constant gross reserves growth rate, let $\eta \equiv B^{+} / B$ denote the constant gross bond growth rate, and let $\mathcal{B} \equiv \theta B / M$ denote the bonds-to-reserves ratio, where $\theta$ denotes the fraction of bonds that FIs use as collateral in the money market. It is assumed that there are positive initial stocks of reserves $M_{0}$ and bonds $B_{0}{ }^{10}$ A stationary equilibrium requires a constant growth rate for the supply of reserves. Furthermore, in any stationary equilibrium the stock of reserves and the stock of bonds must grow at the same rate. In what follows we therefore assume $\gamma=\eta$.

Market clearing in the goods market requires

$$
q_{s}-\int_{0}^{\infty} q_{\varepsilon} d F(\varepsilon)=0
$$

where $q_{s}$ is aggregate production by firms in the goods market.

Market clearing in the money market is affected by the presence of the central bank's standing facilities. To understand their role, let $\rho_{m}^{u}$ denote the rate that would clear the money market in the absence of the standing facilities. This rate is called the unrestricted money market rate. From Lemma 1, the supply and demand of reserves satisfy

$$
\begin{aligned}
S\left(\rho_{m}^{u}\right) & =\int_{0}^{\varepsilon_{1}} p\left(\rho_{m}^{u} / \rho_{d}\right)\left(\varepsilon_{1}-\varepsilon\right) d F(\varepsilon) \\
D\left(\rho_{m}^{u}\right) & =\int_{\varepsilon_{1}}^{\varepsilon_{2}} p\left(\rho_{m}^{u} / \rho_{d}\right)\left(\varepsilon-\varepsilon_{1}\right) d F(\varepsilon)+\int_{\varepsilon_{2}}^{\infty} \rho_{m}^{u} b d F(\varepsilon),
\end{aligned}
$$

respectively, where $\varepsilon_{1}=\frac{m}{p} \frac{\rho_{d}}{\rho_{m}^{u}}$ and $\varepsilon_{2}=\left(\frac{m}{p} \frac{\rho_{d}}{\rho_{m}^{u}}\right)\left(1+\rho_{m}^{u} \frac{\theta b}{m}\right)$. Money market clearing requires $S\left(\rho_{m}^{u}\right)=D\left(\rho_{m}^{u}\right)$, which can be written as follows:

$$
\int_{0}^{\varepsilon_{1}}\left(\varepsilon_{1}-\varepsilon\right) d F(\varepsilon)=\int_{\varepsilon_{1}}^{\varepsilon_{2}}\left(\varepsilon-\varepsilon_{1}\right) d F(\varepsilon)+\int_{\varepsilon_{2}}^{\infty}\left(\varepsilon_{2}-\varepsilon_{1}\right) d F(\varepsilon) .
$$

Suppose (14) yields $\rho_{m}^{u}>\rho_{d}$; i.e., the deposit rate is higher than the unrestricted money market rate. In this case, FIs prefer to deposit reserves at the central bank, which reduces the supply of reserves until $\rho_{m}^{u}=\rho_{d}$. Thus, if $S\left(\rho_{d}\right)>D\left(\rho_{d}\right)$, we must have $\rho_{m}=\rho_{d}$. Along the same lines, suppose (14) yields $\rho_{m}^{u}<\rho_{\ell}$. In this case, FIs prefer to borrow reserves at the central bank's lending facility, which reduces the demand for reserves until $\rho_{m}^{u}=\rho_{\ell}$. Thus, if $S\left(\rho_{\ell}\right)<D\left(\rho_{\ell}\right)$, we must have $\rho_{m}=\rho_{\ell}$. Finally, if $\rho_{d}>\rho_{m}^{u}>\rho_{\ell}$. FIs prefer to trade in the money market, so $\rho_{m}=\rho_{m}^{u}$.

Accordingly, the market-clearing condition can be formulated as follows:

$$
\rho_{m}=\left\{\begin{array}{lll}
\rho_{d} & \text { if } & D\left(\rho_{d}\right)<S\left(\rho_{d}\right) \\
\rho_{\ell} & \text { if } & D\left(\rho_{\ell}\right)>S\left(\rho_{\ell}\right) \\
\rho_{m}^{u} & & \text { otherwise. }
\end{array}\right.
$$

Proposition 2 A symmetric stationary equilibrium with a positive demand for reserves and bonds is a policy $\left(\rho_{d}, \rho_{\ell}\right)$, with endogenous variables $\left(\rho, \rho_{m}, \varepsilon_{1}, \varepsilon_{2}\right)$ satisfying the money market clearing condition (15) and

$$
\begin{aligned}
\rho_{d} \gamma / \beta & =\int_{0}^{\varepsilon_{2}}\left(\rho_{d} / \rho_{m}\right) d F(\varepsilon)+\int_{\varepsilon_{2}}^{\infty}\left(\rho_{d} / \rho_{m}\right)\left(\varepsilon / \varepsilon_{2}\right) d F(\varepsilon) \\
\rho \gamma / \beta & =\int_{0}^{\varepsilon_{2}} d F(\varepsilon)+\int_{\varepsilon_{2}}^{\infty}\left(\varepsilon / \varepsilon_{2}\right) d F(\varepsilon) \\
\varepsilon_{2} & =\varepsilon_{1}\left(1+\rho_{m} \mathcal{B}\right) .
\end{aligned}
$$

Proof of Proposition 2. See the Web Appendix.

\footnotetext{
${ }^{10}$ Since the assets are nominal objects, the government and the central bank can start the economy off with one-time injections of cash $M_{0}$ and bonds $B_{0}$.
} 
Equation (16) is obtained from the choice of reserves holdings (5). Equation (17) is obtained from (5) and (6); in any equilibrium with a strictly positive demand for reserves and bonds, $\rho V_{M}^{m}(m, b)=V_{M}^{b}(m, b)$. Then, this arbitrage equation is used to derive (17). Finally, equation (18) is derived from the budget constraints of the FIs. Note that in any equilibrium $\rho_{m}=\rho$. This result follows from (16) and (17). Note also that once one has derived the endogenous variables $\left(\rho, \rho_{m}, \varepsilon_{1}, \varepsilon_{2}\right)$, one can easily solve for all other endogenous variables. For example, the consumption quantities $q_{\varepsilon}$ and the real value of reserves $m / p$ in the goods market are derived in Lemma 1 .

\section{Quantitative analysis}

The model is calibrated to the Swiss franc repo market because of its outstanding data quality. However, our findings apply to monetary policy implementation and money market functioning in general. We perform in-sample and out-ofsample tests to assess the fit of the model. For the calibration, the distinction between full fiscal support and no fiscal support plays no role. Therefore, we discuss this topic in Sections 4 and 5 and only mention it in this section when it is relevant.

The quantitative analysis covers the period from 2005 to 2013 including 107,517 overnight trades. Standard to the literature, month-end data as well as end-of-maintenance period data are excluded (see, for instance, Thornton, 2006). The model is calibrated to the moments of 244 trading days in the sample period which ranges from 3 January 2005 to 15 December 2005 (baseline sample). In the baseline sample, the SNB kept its monetary policy unchanged. The average overnight rate $\left(i_{m}^{e}\right)$ was $0.63 \%$, the average lending rate $\left(i_{\ell}^{e}\right)$ was $2.61 \%$, and the remuneration of reserves $\left(i_{d}^{e}\right)$ was $0 \%$. The average overnight turnover amounted to CHF $2.77 \mathrm{bn}$. Finally, the average stock of reserves was CHF $5.62 \mathrm{bn}$.

\subsection{Calibration}

For the calibration, we choose the model period as one day. The function $u(q)$ is $\log (q)$ and the liquidity shock $\varepsilon$ is $\log$-normally distributed with mean $\mu$ and standard deviation $\sigma$. The parameters to be identified are (i) the preference parameter $\beta$; (ii) the consumer price index (CPI) inflation $\gamma$; (iii) the policy parameters $\rho_{\ell}$ and $\rho_{d}$; (iv) the bond-toreserves ratio $\mathcal{B}$; and $(\mathrm{v})$ the moments $\mu$ and $\sigma$. All data sources are provided in the Web Appendix. Table 1 reports the identification restrictions and the identified parameter values.

We set $\beta=\left(1+r^{e}\right)^{-1}=0.99812$ so that the model's real interest rate matches the average real interest rate in the data, $r^{e}=0.00188$ which is the difference between one-year Swiss treasury bond yields and CPI inflation. We set $\rho_{\ell}=\left(1+i_{\ell}^{e}\right)^{-1}=0.97454$ and $\rho_{d}=\left(1+i_{d}^{e}\right)^{-1}=1$ to replicate the average lending and deposit rate. To match the average CPI inflation, we set $\gamma=\gamma^{e}=1.01173$. We normalized $M=1$. Furthermore, $\mu=1$, since the numerical analysis shows that $\mu$ is not relevant for our results.

The targets discussed above allow us to calibrate all parameters except the bonds-to-reserves ratio, $\mathcal{B}$, and the standard deviation, $\sigma$. Both are determined by simultaneously matching the average money market rate, $i_{m}^{e}$, and the average turnover-to-reserves ratio, $v^{e}$, by minimizing the weighting function $\min _{\sigma, \mathcal{B}} \omega\left(\left|i_{m}-i_{m}^{e}\right|\right)+(1-\omega)\left(\left|v-v^{e}\right|\right)$ with $\omega=0.5$. We calculate the model's turnover-to-reserves ratio $v$ as follows: Lemma 1 is used to derive $z_{\varepsilon} / M$ for each $\varepsilon$. We then calculate the integral $\int_{\varepsilon_{1}}^{\infty}\left(z_{\varepsilon} / M\right) d F(\varepsilon)$.

Table 1: Calibration targets ${ }^{\mathrm{a}}$

\begin{tabular}{llll}
\hline \hline Parameter & Target description & Parameter value & Target value \\
\hline$\beta$ & Average real interest rate $r^{e}$ & 0.99812 & 0.00188 \\
$\gamma$ & Average inflation rate $\gamma^{e}$ & 1.01173 & 1.01173 \\
$\rho_{\ell}$ & Average lending rate $i_{\ell}^{e}$ & 0.02617 & 0.02617 \\
$\rho_{d}$ & Average deposit rate $i_{d}^{e}$ & 0 & 0 \\
$\mathcal{B}$ & Average money market rate $i_{m}^{e}$ & 0.03934 & 0.00631 \\
$\sigma$ & Average turnover-to-reserves ratio $v^{e}$ & 0.04747 & 0.01548 \\
$\mu, M$ & Normalized & 1 & 1 \\
\hline
\end{tabular}

\footnotetext{
${ }^{\mathrm{a}}$ Table 1 displays the parameters, the targets and their calibrated values. The discount factor $\beta$, the inflation rate $\gamma$, the central bank lending rate $\rho_{\ell}$, and the central bank deposit rate $\rho_{d}$ can be set equal to their target. The bonds-to-reserves ratio $\mathcal{B}$ and the the standard deviation $\sigma$ are determined by simultaneously matching $i_{m}^{e}$ and $v^{e}$. $\mu$ and $M$ are normalized to one.
}

Finally, $\mathcal{B}=\theta B / M$ and so we are left to calibrate $\theta$. Recall that the government issues one-period bonds $B_{G}=$ $\bar{B}_{g}+B_{g}$ that are either held by the central bank, $\bar{B}_{g}$, or the FIs, $B_{g}$. Accordingly, $B_{G} / M$ is set to 16.48755 to replicate 
the ratio of outstanding Swiss government bonds to reserves. Furthermore, $\bar{B}_{g} / M$ is set to 0.51465 to match the SNB's holdings of Swiss government bonds in the baseline calibration period. Then, $B_{g} / M=B_{G} / M-\bar{B}_{g} / M=15.97290$. Since no central bank bills $B_{c}$ were outstanding during the baseline calibration period $B=B_{g}+B_{c}=B_{g}$ implying that $B / M=B_{g} / M=15.97290$. Hence, from $\mathcal{B}=\theta B / M, \theta=0.00246$. This number indicates that FIs only commit a small quantity of their government bond holdings as collateral to trade in the money market.

\section{$3.2 \quad$ In-sample fit}

To assess the model's in-sample fit, the idiosyncratic liquidity shocks are drawn from a log-normal distribution with the calibrated moments $\mu$ and $\sigma$. The liquidity shocks are assumed to be unexpected and we are abstracting from aggregate shocks. To generate a sequence of $i_{m}^{t}$ and $v^{t}$, the sampling exercise is repeated for $t=1, \ldots, T$ periods. The exact simulation procedure is described in the Web Appendix.

TABle 2: Empirical and simulated MOMEnts ${ }^{\mathrm{a}}$

\begin{tabular}{|c|c|c|c|c|}
\hline & Empirical & & Simulate & \\
\hline$M=1$ (in-sample) & Mean & STD & Mean & STD \\
\hline Money market rate $i_{m}$ & 0.00631 & 0.00064 & 0.00633 & 0.00086 \\
\hline Turnover-to-reserves ratio $v$ & 0.01548 & 0.00512 & 0.01547 & 0.00015 \\
\hline$M=66$ (out-of-sample) & Mean & STD & Mean & STD \\
\hline Money market rate $i_{m}$ & 0 & 0.00017 & 0 & 0 \\
\hline Turnover-to-reserves ratio $v$ & 0.00018 & 0.00014 & 0.00024 & 0 \\
\hline
\end{tabular}

Table 2 displays empirical and simulated means and the standard deviations for $i_{m}$ and $v$ for two cases: in-sample $(M=1)$ and out-of-sample ( $M=66$, discussed in the following subsection). The in-sample comparison shows that the model fits the average $i_{m}^{e}$ and $v^{e}$ as well as the standard deviation of $i_{m}^{e}$ very well. In contrast, the standard deviation of $v$ is too low. The reason is the homogenous collateral constraint: In our model all FIs enter the money market with the same collateral and reserves holdings, and so the collateral constraint is binding at the same value. In practice, FIs are heterogeneous with regard to their collateral and reserves holdings and their collateral constraints are binding at different values.

Figure 2 shows the empirical and simulated distributions of $i_{m}, v$ and the borrowing and lending volumes. The left-hand-side panel in the first row shows the histogram of the simulated and the empirical money market rates in the baseline calibration period. The comparison suggests that the model is able to match the empirical distribution of $i_{m}$ well. In the data, there is a larger concentration of interest rates around the mean than in our simulation.

The distributions of the simulated and empirical turnover-to-reserves ratios are displayed in the right-hand-side panel of the first row of Figure 2. There is a large concentration of the simulated turnover-to-reserve ratio at $v=0.015$. The reason is the collateral constraint as discussed above.

The second row of Figure 2 suggests that the model can fit the empirical distribution of lending volumes quite well. The frequency is decreasing in size and the model-generated data is able to match this feature. However, the model generates insufficiently small lending volumes compared to the empirical data. As discussed before, there is a large concentration of the simulated borrowing volumes at 0.04 , and the reason is again that the collateral constraint binds at this value for all FIs in our model. 
FiguRE 2: IN-SAMPLE-DISTRIBUtion ANALYSIS ${ }^{\mathrm{a}}$

Money market rate and turnover-to-reserves ratio distributions
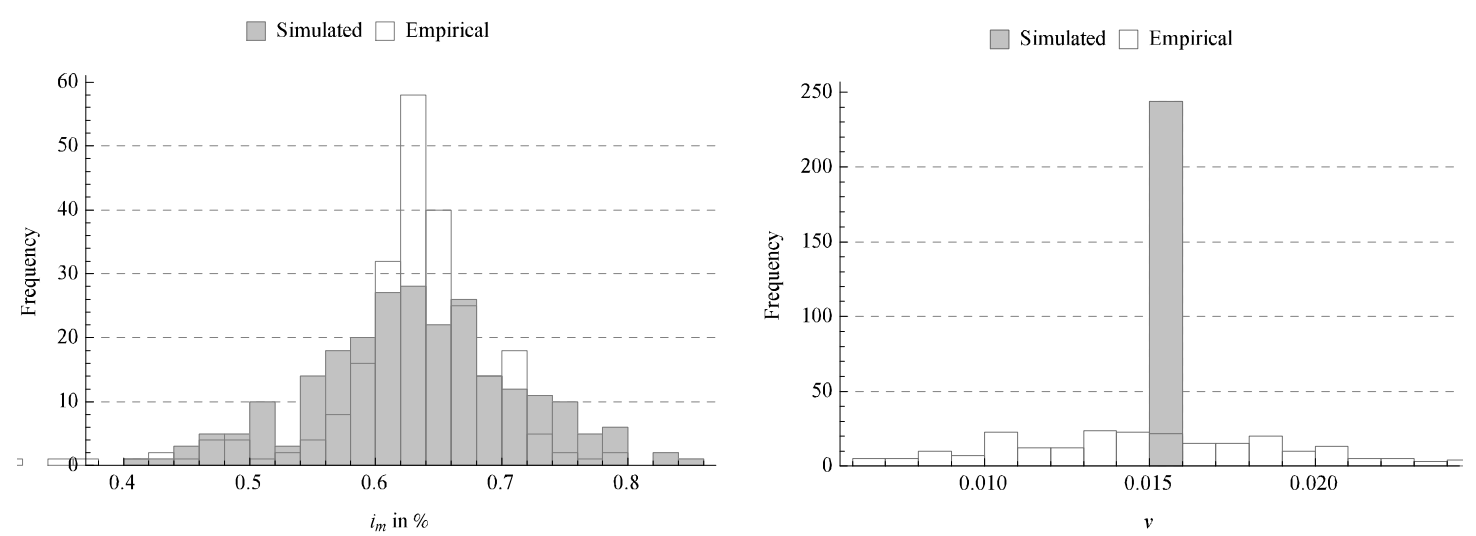

Lending and borrowing volume distributions
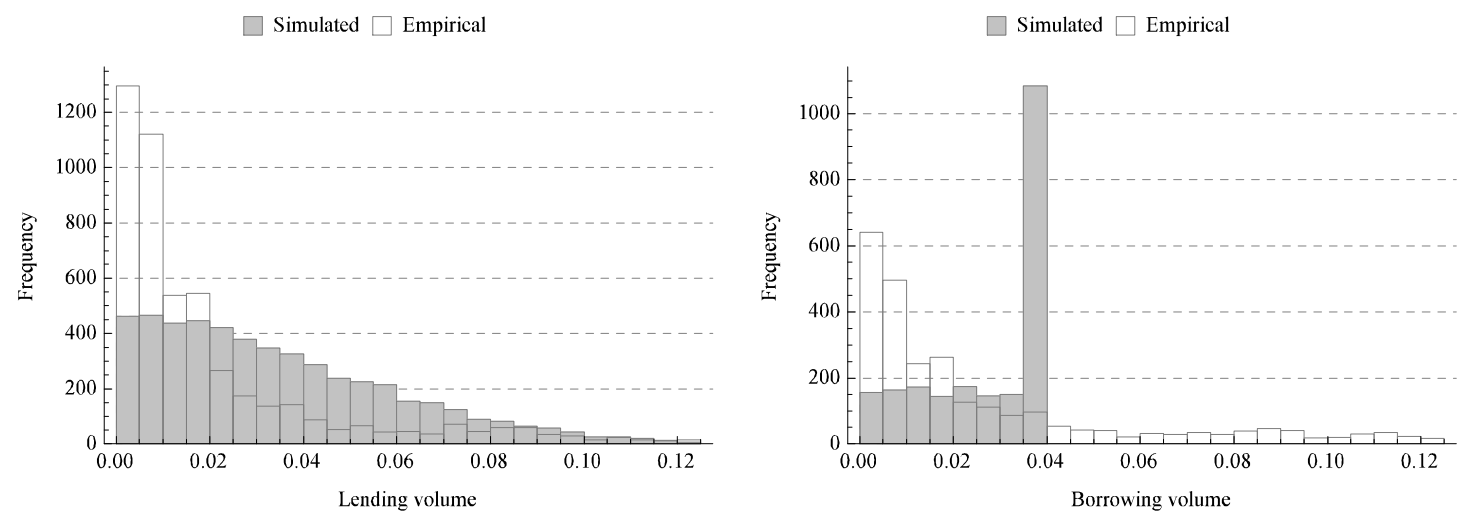

${ }^{\mathrm{a}}$ The panels in the first row show the histograms of the simulated and empirical money market rates $i_{m}$ and the turnover-to-reserves ratios $v$ in the baseline calibration period. The panels in the second row compare the simulated and the empirical lending and borrowing volume sizes for the same period.

\subsection{Out-of-sample fit}

To assess the model's out-of-sample fit, we conduct two experiments. The first experiment considers small reserve supply shocks in a structural liquidity deficit environment. In the second experiment, the central bank increases the quantity of reserves by a large amount and, thereby, creates a structural liquidity surplus environment. Although we follow the SNB's experience with these two experiments, our findings apply to all major central banks that implemented similar policies and operated in comparable environments. Note that these experiments are tests of how our model performs out-of-sample, as we do not recalibrate the model.

Structural liquidity deficit: scarcity of reserves. Prior to the crisis, all major central banks created an environment where the banking system was kept short of reserves, a so-called structural liquidity deficit. In such an environment, the central bank just provides sufficient reserves such that FIs are able to meet their minimum reserve requirements. Consequently, reserves are scarce, and the central bank can achieve the desired interest rate by simply changing the stock of reserves by a small amount. To provide the correct amount of reserves, the central bank forecasts FIs' demand for reserves, taking into account exogenous factors such as notes in circulation or the government's reserve balances. When the central bank underestimated (overestimated) the demand for reserves, the money market rate increased above (decreased below) the target rate.

In what follows, we consider a situation in which the central bank has unexpectedly provided either an excess or an insufficient reserves to the banking system. The focus is on the relationship between $M$ and $i_{m}$, and the experiment is called temporary $M$-shock. Note that a temporary shock does not affect the price of reserves $\phi$ in the settlement market, since $\phi$ is a forward-looking variable: that is, it is determined by future monetary conditions, only. Thus, for a temporary shock, we keep $\phi$ at the calibrated value. 
Our simulation method is described in the first paragraph of Section 3.2. We set $T=40$. A box-plot representation is chosen to illustrate the simulated data. The box-plots displayed in Figure 3 and in all the following figures provide the following information: First, the means of the simulated $i_{m}^{t}$ and $v^{t}$ are indicated by the black horizontal line in the blue area. Second, the width between the 25 th and the 75 th percentile is represented by the blue area. Third, the minimum and maximum values are indicated by the vertical lines at the extremes of the box-plot.

FigURE 3: $M$-shocks ${ }^{\mathrm{a}}$
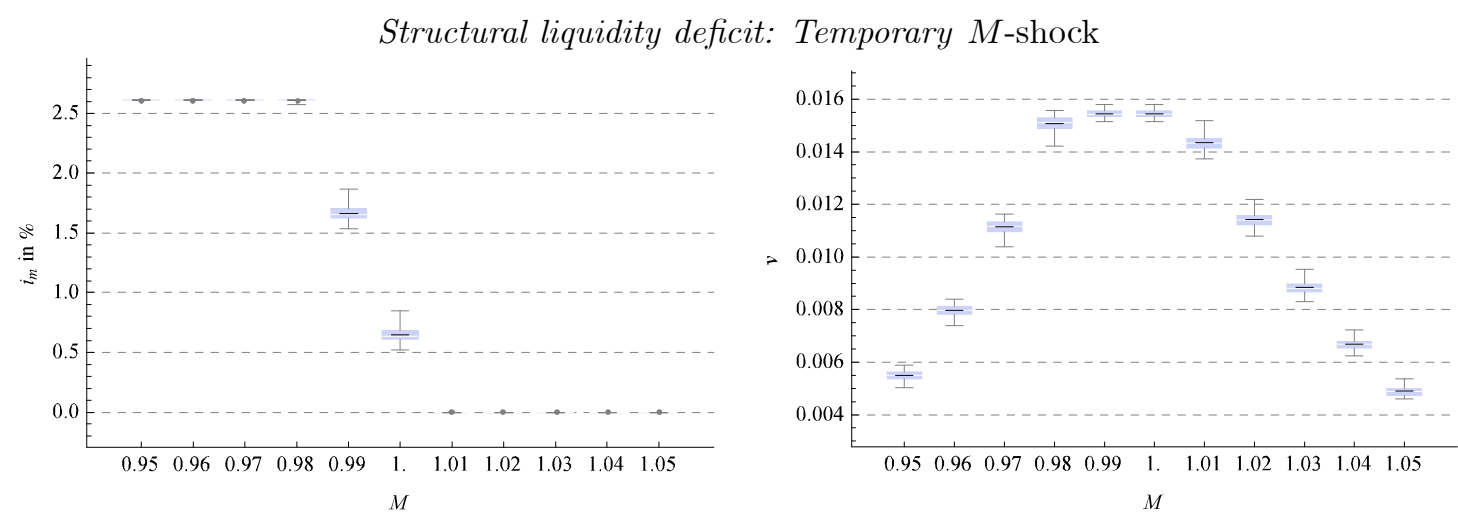

Structural liquidity surplus: Permanent $M$-shock
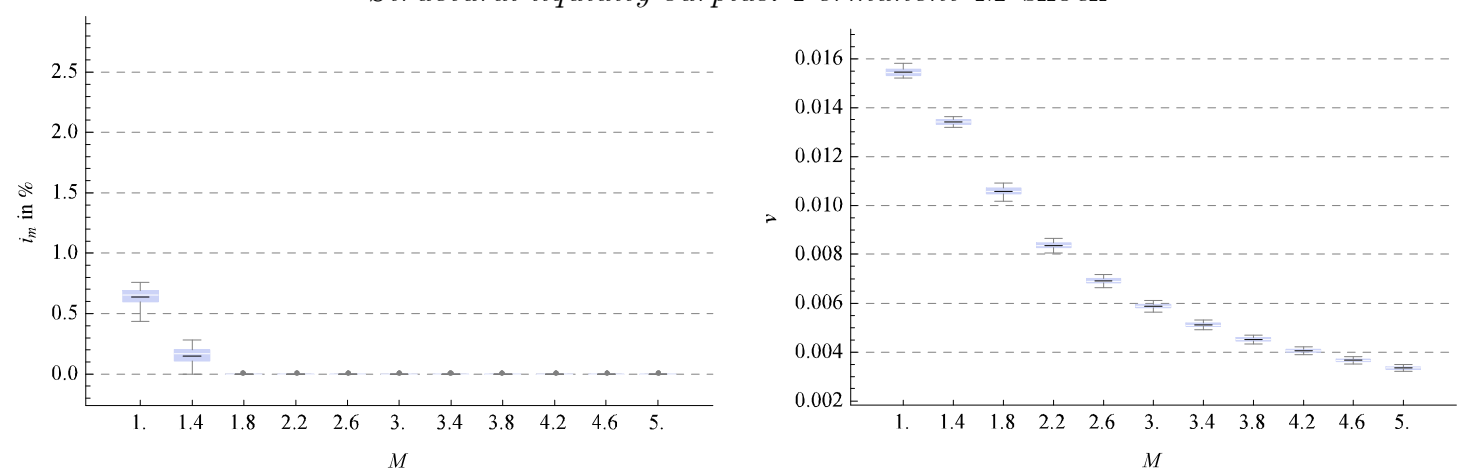

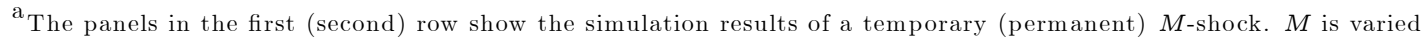
by $+/-5 \%$ from the calibrated value $M=1$ ( $M$ is increased from $M=1$ to $M=66$ ). The left-hand-side panels show the impact on the money market rate $i_{m}$ and the right-hand-side panels on the turnover-to-reserves ratio $v$.

The simulation results of a temporary $M$-shock are shown in the first row of Figure 3 . They are based on a variation of $M$ by $+/-5 \%$ from the calibrated value $M=1 .^{11}$ The panel on the left-hand side displays the effect on $i_{m}$, whereas the panel on the right-hand side displays the effect on $v$. The simulation generates the typical relationship between $M$ and $i_{m}$ : a temporary increase in the stock of reserves $M$ reduces the demand for reserves and increases the supply of reserves in the money market. ${ }^{12}$ Consequently, $i_{m}$ decreases and eventually reaches the deposit rate. In contrast, a decrease in $M$ increases the demand for reserves and decreases the supply of reserves. As a result, $i_{m}$ increases and ultimately reaches the lending rate.

At the calibrated value $M=1, i_{m}$ reacts highly elastically to changes in $M$ : a change in $M$ of $1 \%$ is associated with a change in $i_{m}$ of $81 \%$. This is in line with empirical studies that estimate elasticities for the demand for reserves. For instance, Kraenzlin and Schlegel (2012) estimate that a change in $M$ of $5 \%$ at the end of the minimum reserve requirement period is associated with a change in $i_{m}$ of up to 60 basis points (applied to our simulation, this is an interest rate change of approximately 100\%). As $M$ moves away from the calibrated value, $i_{m}$ becomes less elastic. Eventually, due to the presence of the standing facilities, the elasticity drops to zero.

The turnover-to-reserves ratio $v$ is increasing in $M$ if $M<1$ and decreasing if $M \geq 1$. If $M$ is small, there is excess demand for reserves, $i_{m}$ is at $i_{\ell}$, and FIs borrow at the lending facility. These borrowed reserves are not included in the calculation of $v$, since the turnover-to-reserves ratio represents the traded volume in the money market, only. The same is true if $M$ is large. In this case, there is an excess supply of reserves, $i_{m}$ is at $i_{d}$, and the excess supply of reserves is absorbed by the deposit facility.

\footnotetext{
${ }^{11}$ For each $M$, we use the same random sample of liquidity shocks.

${ }^{12}$ We provide figures of how the demand and the supply curves of reserves are affected by changes in $M$ in the Web Appendix.
} 
Structural liquidity surplus: large excess reserves. In response to the financial crisis of 2007/08 and the subsequent sovereign debt crisis, all major central banks decreased interest rates to historically low levels and created large excess reserves via asset or foreign currency purchases (QE). In the case of Switzerland, the SNB increased reserves via foreign exchange purchases from CHF 5.62 bn in 2005 to CHF 370 bn in 2013 (a factor of 66). ${ }^{13}$ As a result, the banking system is in a structural liquidity surplus with FIs holding large excess reserves, money market interest rates are at $i_{d}$, and money market activity has collapsed as shown in Figures F.1 and F.2 in the Web Appendix.

In the following, we study a permanent and large increase in the stock of reserves from $M=1$ to $M=66 .{ }^{14} \mathrm{In}$ contrast to the temporary $M$-shock, we let the price of reserves $\phi$ adjust to its new equilibrium value. Note that although all prices are fully flexible in our model, a change in $M$ is not neutral. The reason for the non-neutrality is the collateral constraint. Note also that this experiment is conducted under full fiscal support. That is, as $M$ increases, we assume that the real transfer $\phi T$ endogenously adjusts to satisfy the consolidated government budget constraint (3). In this experiment, we keep the inflation rate constant at the calibrated value. ${ }^{15}$ As before, we study the effects on the moments and the distributions of $i_{m}$ and $v$, as well as the distributions of the borrowing and lending volumes.

Table 2 reports the money market rate and the turnover-to-reserves ratio for $M=66$. The model predicts a money market rate of zero and a turnover-to-reserves ratio of 0.00024. Both simulated values are very close to the empirical counterparts. The dynamics of a permanent increase of $M$ is also shown in Figure 3. It matches the stylized facts shown Figures F.1 and F.2 in the Web Appendix).

Figure 4: OUt-OF-SAMPle Distribution ANALYSis ${ }^{\mathrm{a}}$

Money market rate and turnover-to-reserves ratio distributions
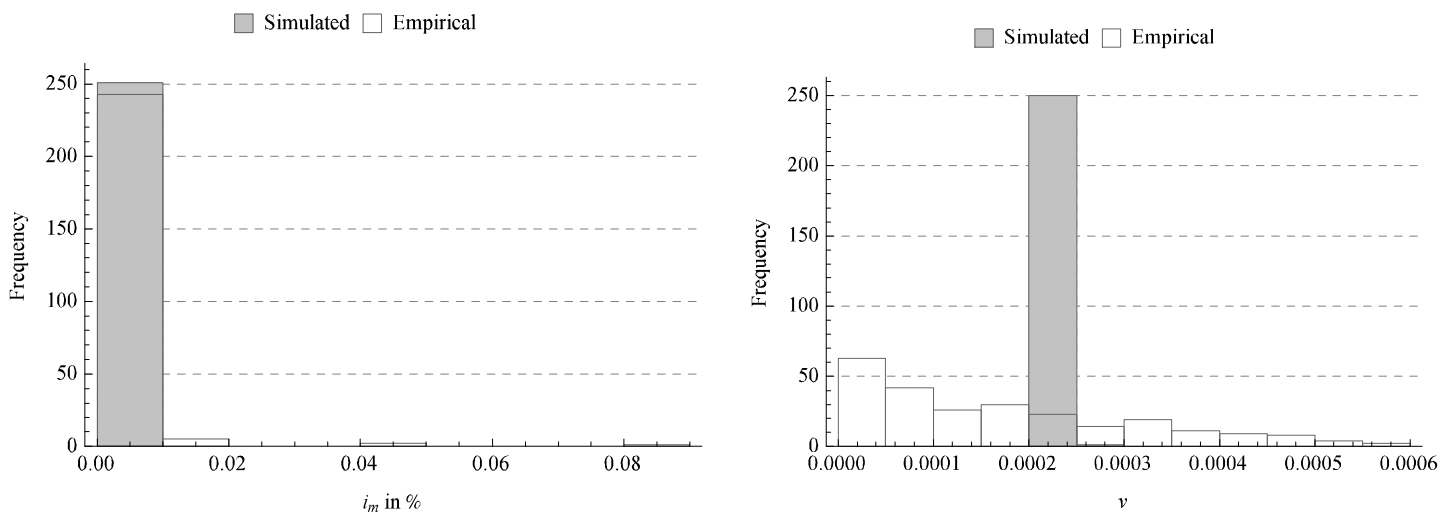

Lending and borrowing volume distributions
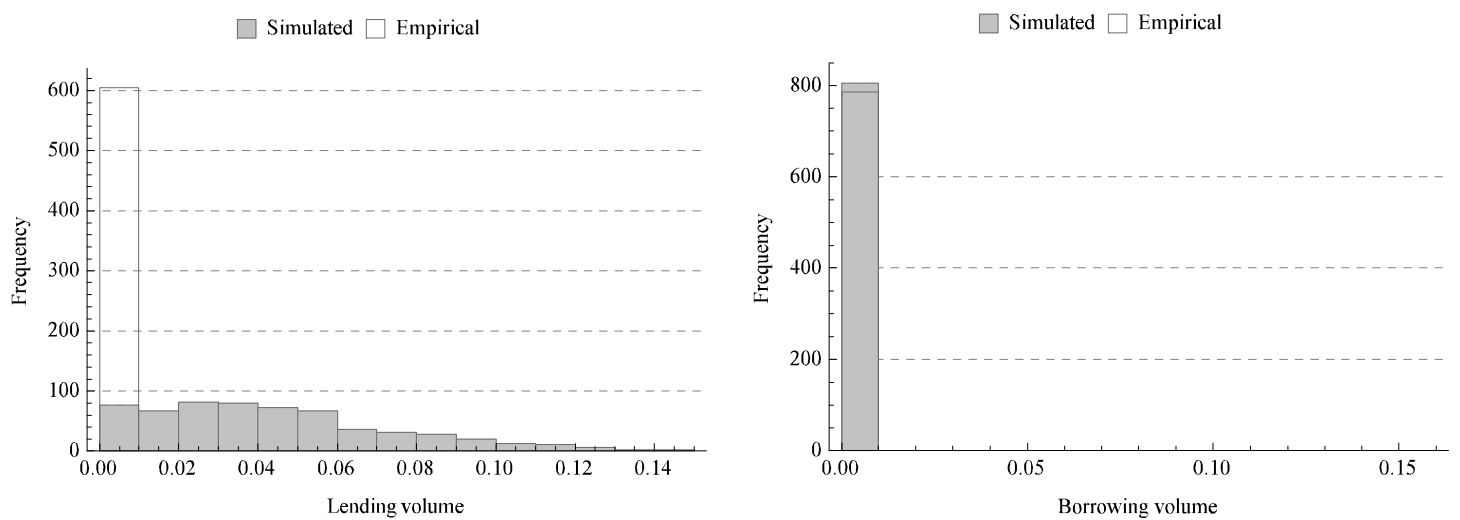

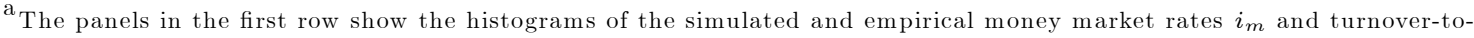
reserves ratios $v$ in 2013. The panels in the second row compare the simulated and the empirical lending and borrowing volume sizes in 2013 .

\footnotetext{
${ }^{13}$ The SNB continued to be active in the foreign currency market after 2013. As a result, reserves continued to increase and stood at CHF 580 bn as of mid-2017.

${ }^{14} \mathrm{An}$ increase in reserves via QE is a permanent $M$-shock, since the central bank's counterparties are usually non-FIs. Therefore, we study a large and permanent increase in the stock of reserves $M$ holding FIs' stock of collateral $B$ constant (see Benford et al. 2009 for a reference).

${ }^{15}$ We also conducted the same experiment under no fiscal support, where $\phi T$ is kept constant and the inflation rate $\gamma$ adjusts to satisfy (3). For this experiment, we find that the inflation rate increases to $8 \%$, which has not been observed in any country that has a major central bank. Therefore, we present this experiment under full fiscal suppport, only.
} 
The first row of Figure 4 shows the empirical and simulated distributions of $i_{m}$ and $v$. The left-hand-side panel suggests that the model is able to fit the empirical distribution of $i_{m}$ well. ${ }^{16}$ In contrast, the model again fails to match the empirical distribution of $v$, shown in the right-hand side panel. Again, this is due to the homogeneous collateral constraint. The second row of Figure 4 shows the distributions of lending and borrowing volumes. In the case of the lending volumes, the model simulations predict a less positively skewed distribution, suggesting that the lending volumes generated by the model are more variable than the empirical observations. In the case of the borrowing volumes, the simulated distribution fits the empirical data quite well: FIs are only willing to borrow small volumes because of excess reserves.

\section{Exit strategies}

The current monetary policy environment is characterized by large excess reserves. As a result, the money market rate is at the deposit rate, and the trading activity is close to zero. Central bankers and academics currently discuss how monetary policy should be implemented, going forward. The term 'exit strategies' refers to policies that allow central banks to control short-term interest rates in an environment with large excess reserves and three strategies are widely discussed: IOR, absorbing reserves using term deposits, central bank bills, or reverse repos, and reducing the balance sheet by selling assets purchased under QE. For each of these strategies, the focus of our attention will be on the implications for welfare, their effectiveness in controlling the money market rate, how money market trading activity is affected, and its effect on inflation and transfers. We also calculate the required interest payments by the central bank to the FIs in order to implement a certain interest rate target.

In the simulation of exit strategies, we will use the consolidated budget constraint to analyze the impact of the various policies on the real transfer $\phi T$ because $T$ affects welfare. $T_{c}$ is key here because it maps directly into $T$, through (1). The analysis of the exit strategies is conducted under full fiscal support and no fiscal support discussed in Section 2.2. For that purpose, it is useful to rewrite the consolidated budget constraint (3) as follows (see Web Appendix):

$$
\phi T=\frac{\rho \varepsilon_{1}}{\beta}\left[\gamma-1 / \rho_{d}+(B / M)(\rho \gamma-1)+\left(1 / \rho_{\ell}-1 / \rho_{d}\right) L / M\right] .
$$

Under full fiscal support, we assume that the real transfer to the public $\phi T$ adjusts endogenously to satisfy the consolidated government budget constraint (19) and that the rate of inflation $\gamma$ is kept constant at the calibrated value. For example, when changing $\rho_{d}$, we take into account the direct effect of $\rho_{d}$ on $\phi T$ and the general equilibrium effects of $\rho_{d}$ on $\phi T$ via changes in $\rho$ and $\varepsilon_{1}$. Under no fiscal support, we assume that the real transfer $\phi T$ is kept constant at the calibrated value $\overline{\phi T}$, and that $\gamma$ adjusts endogenously. All simulations in this section have the same initial conditions: The initial stock of reserves is $M=66$, which corresponds to the average stock of reserves in 2013, and the initial inflation rate is at the calibrated value $\gamma=1.01$. Using these values, we find that the transfer that is needed to satisfy the consolidated government budget constraint (19) is $\overline{\phi T}=0.04$.

Recall that $\phi T$ is a lump-sum transfer. Thus, full fiscal support implicitly assumes that any change in policy results in a change of a non-distortionary tax. In contrast, under no fiscal support, $\phi T$ is kept at the calibrated value, and the inflation rate, which is a distortionary tax, adjusts to a change in policy to satisfy (19).

\subsection{Interest on reserves}

With IOR the central bank remunerates reserves at rate $i_{d}$, which imposes a floor for the overnight money market rate: No FI would lend to other counterparties at a rate below $i_{d} \cdot{ }^{17}$ In an environment with large excess reserves, $i_{m}$ will be equal to $i_{d}$. For that reason, this method of implementing monetary policy is referred to as a floor system. IOR is simulated by increasing $i_{d}$ and the simulation results are displayed in Figure 5 .

Money market rate and turnover. There is no qualitative discrepancy for full fiscal support and no fiscal support: In both cases, the money market rate satisfies $i_{m}=i_{d}$ for all values of $i_{d}$, and the trading activity in the money market

\footnotetext{
${ }^{16}$ Note that a considerable amount of transactions was executed at negative interest rates in 2013. The motivation was to acquire certain types of collateral used in other money market segments. As this motivation is not reflected in our model, negative interest rates are set to zero to make the model's predictions comparable to the empirical observations.

${ }^{17}$ The IOR might represent a binding floor or should at least serve as a magnet for the overnight money market rate. This depends on the degree of segmentation and competition in the money market, as well as on the central bank's access policy to a reserve account, and hence access to earning the IOR. See Bech and Klee (2011) for an analysis of the federal funds market and Jackson and Sim (2013) for a study of the UK case. For an analysis of the impact of central banks' access policies, see Kraenzlin and Nellen (2015).
} 
is close to zero. This shows that the central bank can control $i_{m}$ with perfect precision without changing the stock of reserves in the economy.

Inflation, transfer, and welfare. Under full fiscal support, the transfer monotonically decreases from its initial value $\overline{\phi T}$ and eventually becomes negative (a tax). Welfare is increasing and attains the first-best welfare level at $i_{d}=1.36 \%$. The reason is that the interest payments are financed with a non-distortionary tax and that paying IOR is welfare improving. This finding is related to the Friedman rule which requires that the opportunity cost of holding reserves should be zero. Here, this is achieved by paying IOR.

Under no fiscal support, inflation increases from the calibrated value of $1.17 \%$ to $2.56 \%$. The effect on welfare is slightly negative, and it is impossible to attain the first-best welfare level. The intuition is that the interest payments are financed with a distortionary tax. Thus, while paying IOR is welfare improving, the overall effect is negative because of the distortions created by inflation.

\section{Figure 5: Interest ON RESERVES ${ }^{\mathrm{a}}$}

Full fiscal support and no fiscal support
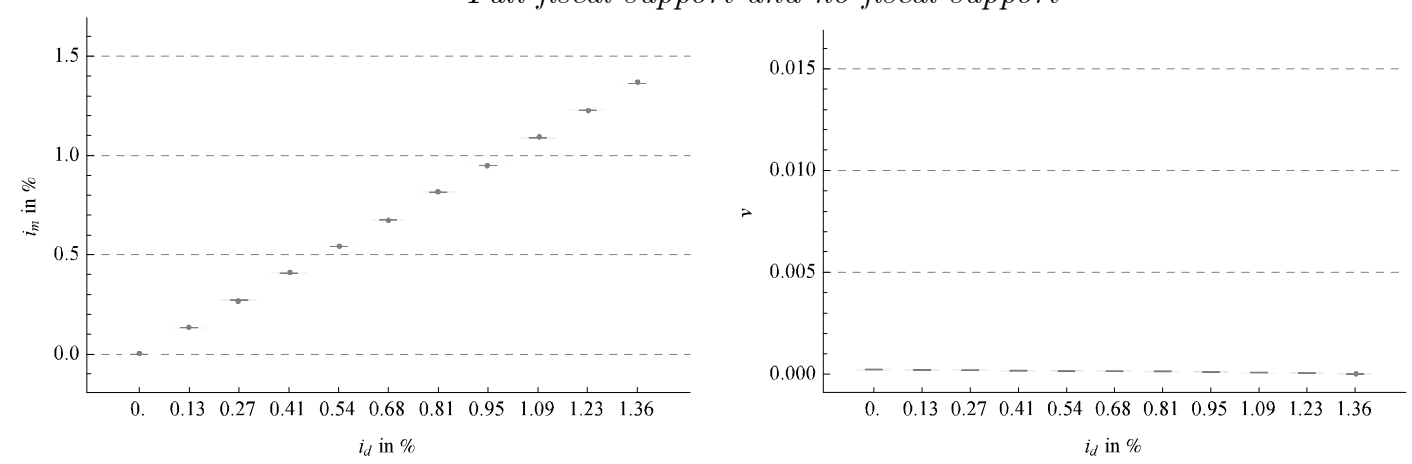

Full fiscal support
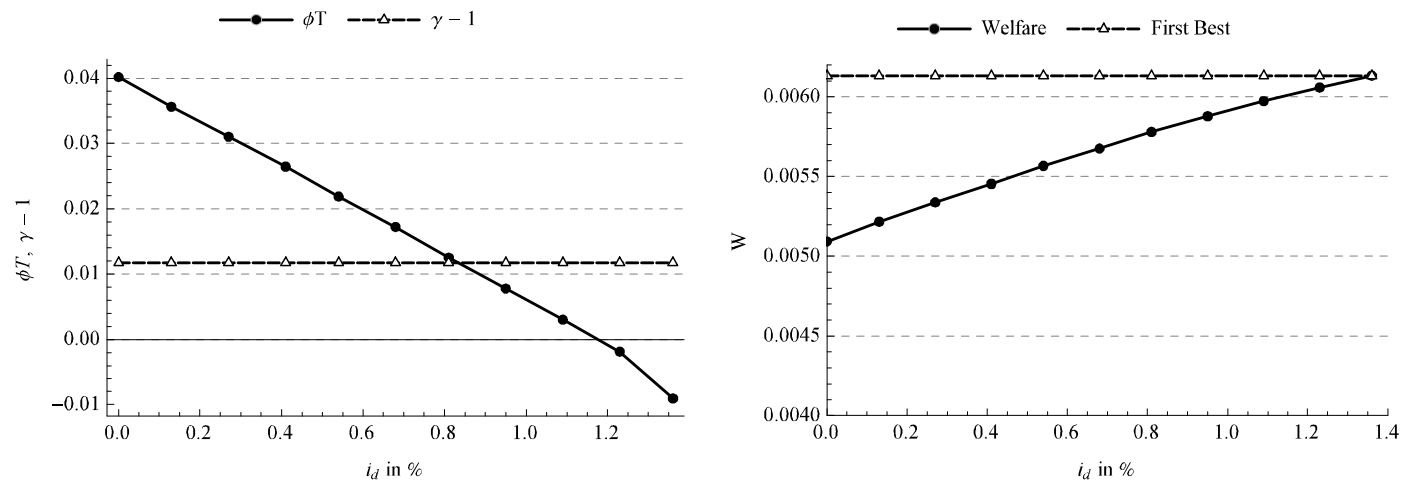

No fiscal support
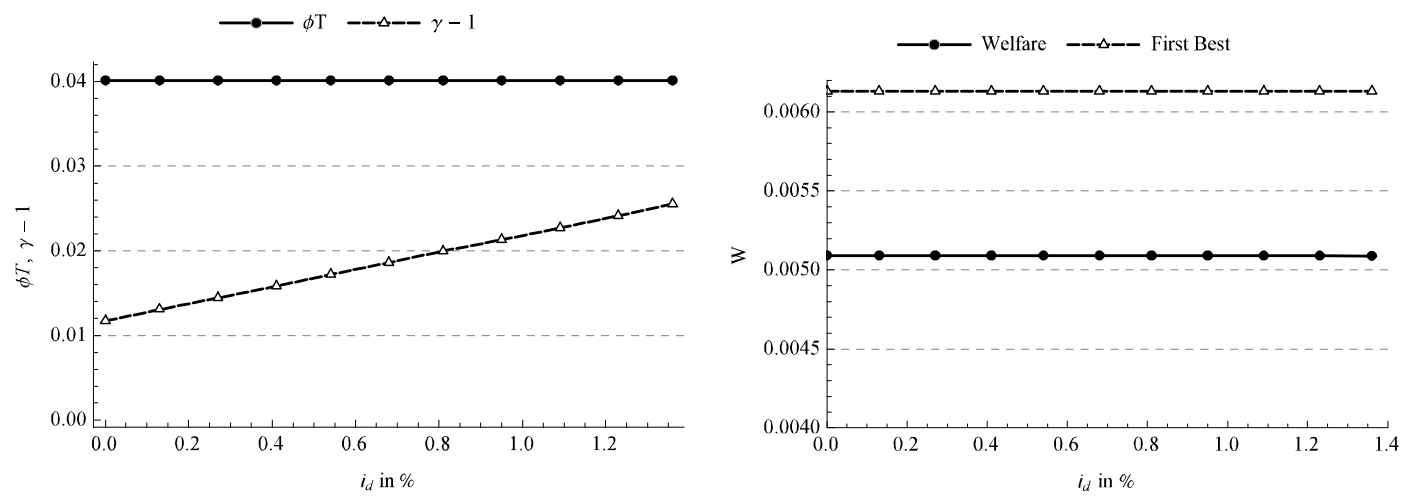

${ }^{\text {a With }} M=66$, the central bank starts to pay IOR. The panels in the first row show the impact on the money market rate $i_{m}$ and the turnover-to-reserves ratio $v$. The panels in the second (third) row show the impact on inflation $\gamma-1$, real transfer $\phi T$, and welfare $W$ under full fiscal support (no fiscal support). 


\subsection{Selling assets and term deposits}

With a term deposit, the central bank issues an IOU and sells it to FIs against reserves. If sufficient reserves are absorbed, they become scarce and the money market rate increases above the deposit rate. Key characteristics of term deposits are that they cannot be traded by FIs and they cannot be used as collateral. The effects of term deposits are, therefore, simulated by reducing reserves from $M=66$ to $M=1$, holding the stock of collateral constant at the calibrated value. In Figure 6 we only show the range between $M=5$ and $M=1$.

With our simulation strategy, absorbing reserves using term deposits is equivalent to selling assets and thus unwinding QE. Both reduce excess reserves by the same amount and we assume this reduction to be permanent. Note that the central bank's balance sheet size remains unchanged with term deposits whereas selling assets reduces the central bank's balance sheet.

Money market rate and turnover. Under full fiscal support, $i_{m}$ remains at $i_{d}$ for $M>1.64$. For $M<1.64, i_{m}$ increases monotonically until it reaches the calibrated money market rate of $0.63 \%$ at $M=1$. Furthermore, $v$ also increases monotonically and eventually reaches the calibrated value at $M=1$. The reason is that absorbing reserves using term deposits or by selling assets reverses QE in our model. ${ }^{18}$

Under no fiscal support, $i_{m}$ only increases above $i_{d}$ for $M<0.64$. Moreover, the money market activity is lower than under full fiscal support. This can be explained as follows: As inflation, and hence the opportunity cost of holding reserves, decreases, FIs demand more reserves in the settlement market (self-insurance) and rely less on the money market to insure against liquidity shocks. This increases the supply of reserves in the money market and decreases the demand for reserves in the money market, which results in a lower money market rate than under full fiscal support. Hence, the money market rate remains at $i_{m}=i_{d}=0 \%$ for values of $M$ where we find a strictly positive rate under full fiscal support. For the same reason, $v$ is lower under no fiscal support than under full fiscal support. Compared to IOR, the control of the money market rate under full fiscal support is less precise, as indicated by the box-plots.

Inflation, transfer, and welfare. Under full fiscal support and in the region of $M$ where $i_{m}=i_{d}=0$, $\phi T$ increases monotonically when $M$ decreases. In this region, the central bank can absorb reserves with term deposits at zero costs, and $\phi T$ increases, because the bond-to-reserves ratio $\mathcal{B}$ is increasing. In the region of $M$, where $i_{m}>i_{d}, \phi T$ can be hump-shaped because now the monetary authority has to pay a strictly positive interest rate to FIs in order to absorb reserves with term deposits. Our simulations indicate that close to $M=1$, the interest cost dominates the benefit of increasing $\mathcal{B}$ and so $\phi T$ is decreasing. With respect to welfare, we find that in the region of $M$ where $i_{m}=i_{d}$, welfare is slightly increasing, because of the increasing bonds-to-reserve ratio. In the region of $M$ where $i_{m}>i_{d}$, welfare is rapidly increasing, because the money market allows FIs to earn interest on 'idle' reserves. Thus, the existence of such a market is strictly welfare-improving, as has been shown in a different context in Berentsen et al. (2007).

Under no fiscal support, we find that inflation is monotonically decreasing, and welfare is monotonically increasing as $M$ decreases. Welfare is increasing because inflation is decreasing. The mechanism at work can be understood by inspecting the consolidated budget constraint (19): A decrease in $M$ increases the bonds-to-reserve ratio $\mathcal{B}$. Endogenous variables such as $\rho$ and $\varepsilon_{1}$ do not adjust enough to satisfy $\phi T=\overline{\phi T}$. Accordingly, $\gamma$ has to decrease until $\phi T=\overline{\phi T}$. We find that the welfare level under no fiscal support is higher than under full fiscal support for any $M \geq 1$. This result is driven by the fact that inflation is lower under no fiscal support.

\footnotetext{
${ }^{18}$ Recall that when we simulated QE, it was under full fiscal support, because the increase in inflation that arises under no fiscal support is not consistent with the data. See our discussion in Section 3.
} 


\section{Figure 6: Selling Assets And term Deposits ${ }^{a}$}

\section{Full fiscal support}
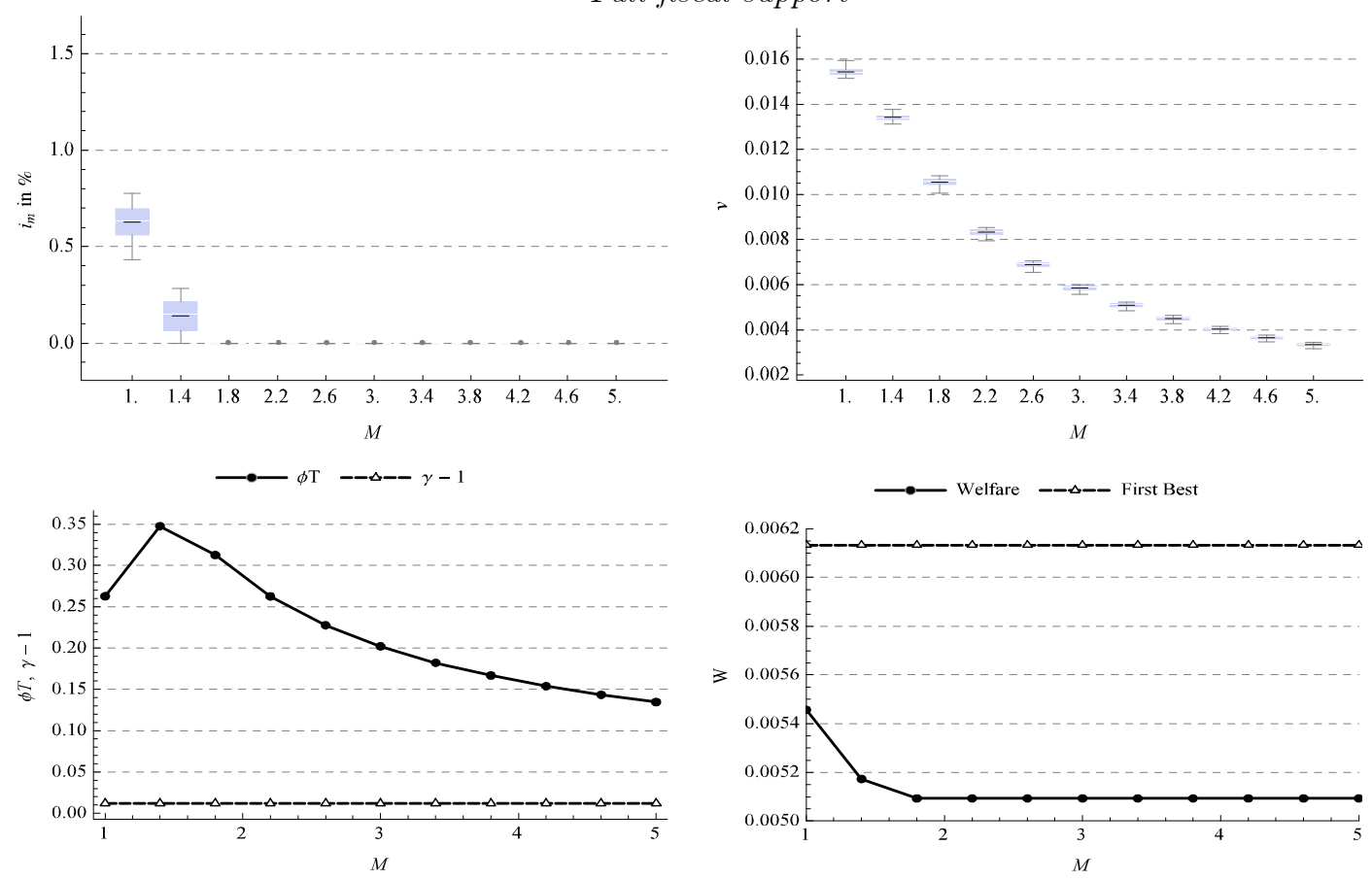

No fiscal support
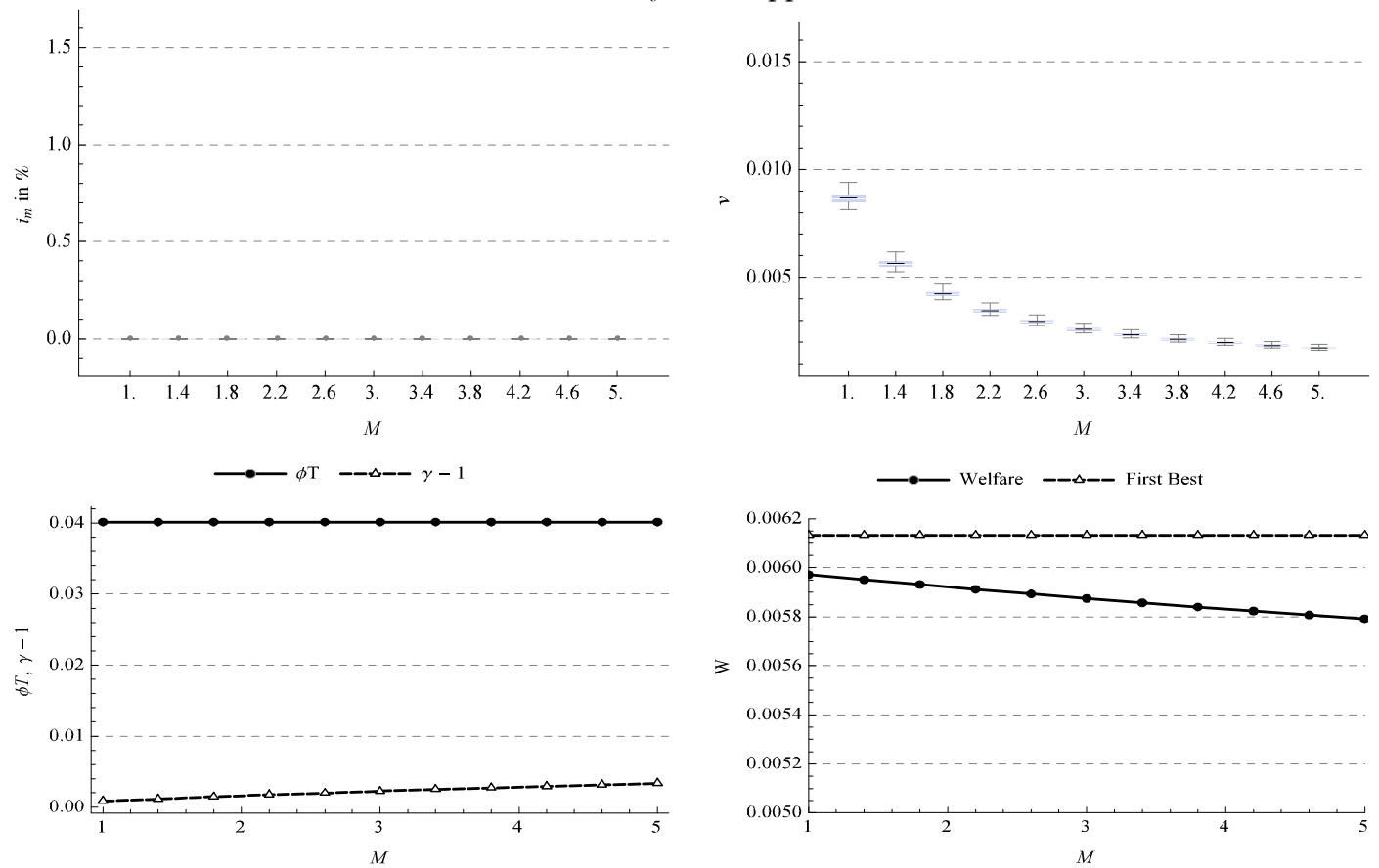

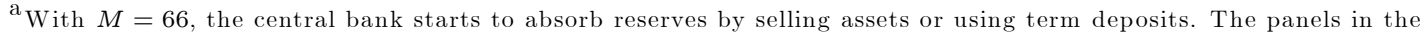
first (third) row show the impact on the money market rate $i_{m}$ and the turnover-to-reserves ratio $v$ under full fiscal support (no fiscal support). The panels in the second (fourth) row show the impact on inflation $\gamma-1$, real transfer $\phi T$, and welfare $W$ under full fiscal support (no fiscal support). 


\subsection{Central bank bills and reverse repos}

With a central bank bill, the monetary authority issues an IOU and sells it to FIs in order to absorb reserves. In contrast to a term deposit, the central bank creates a tradeable security (the IOU) which can be used as collateral by FIs. With a reverse repo, the central bank borrows reserves against collateral, and FIs collateral holdings increase. In practice, central bank bills and repos can also be purchased by non-FIs. Therefore, we need to take a stand to what extent the collateral holdings of FIs increases. In the following simulations, we assume that $M$ decreases from $M=66$ to $M=1$ and that, at the same time, collateral holdings $\theta B$ increase from the calibrated value to $2 \theta B .{ }^{19}$

Money market rate and turnover. Figure 7 shows that under full fiscal support, $i_{m}$ remains at $i_{d}=0$ for $M>2.6$. For $M<2.6, i_{m}$ increases monotonically until it reaches the calibrated money market rate of $0.63 \%$ at $M=1.8$. This shows that central bank bills and reverse repos are more effective than term deposits, since less reserve-absorption is needed if the goal is to increase the money market rate. The reason for this is that an increase in FIs' collateral holdings relaxes the collateral constraint and hence allows them to borrow more in the money market. Thus, for any value of $M$, the demand for reserves in the money market and the interest rate is higher under a policy using central bank bills or reverse repos than under a policy using term deposits. Applying the same logic, the increase in FIs' collateral holdings also leads to more trading activity with central bank bills or reverse repos than with term deposits or IOR.

Under no fiscal support, $i_{m}$ remains at $i_{d}$ for $M>1.12$. For $M<1.12, i_{m}$ increases rapidly until it reaches the calibrated money market rate of $0.63 \%$ at around $M=1.08$. For any value of $M$, there is slightly less trading activity in the money market under no fiscal support than under full fiscal support. The simulation suggests that the control of the money market rate with central bank bills and reverse repos is less precise than with IOR, which is indicated by the box-plots.

Inflation, transfer, and welfare. Under full fiscal support, $\phi T$ increases as $M$ decreases in the region where $i_{m}=$ $i_{d}=0$. In the region where $i_{m}>i_{d}, \phi T$ decreases because the interest payments are financed by a reduction of the transfer. In the region where $i_{m}=i_{d}, W$ is flat, and in the region where $i_{m}>i_{d}, W$ increases because paying IOR financed in a non-distortionary way - decreases the opportunity cost of holding reserves and is thus welfare-improving.

Under no fiscal support, inflation is first decreasing as $M$ decreases and then starts to rapidly increase after $M=1.12$. Welfare mimics this result. It is first increasing and then rapidly decreasing. The reason is that the decrease of inflation decreases the opportunity cost of holding reserves. However, ultimately, the money market rate moves beyond the optimal value that is described by the Friedman rule.

\footnotetext{
${ }^{19}$ The SNB has the legal basis for issuing central bank bills as an instrument to absorb excess reserves. This is not the case, for instance,

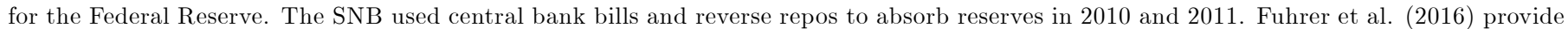

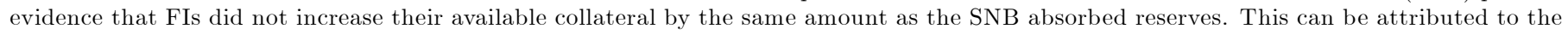
fact that a considerable fraction of outstanding central bank bills were purchased by non-FIs.
} 
Figure 7: Central BAnK Bills And Reverse Repos ${ }^{\mathrm{a}}$
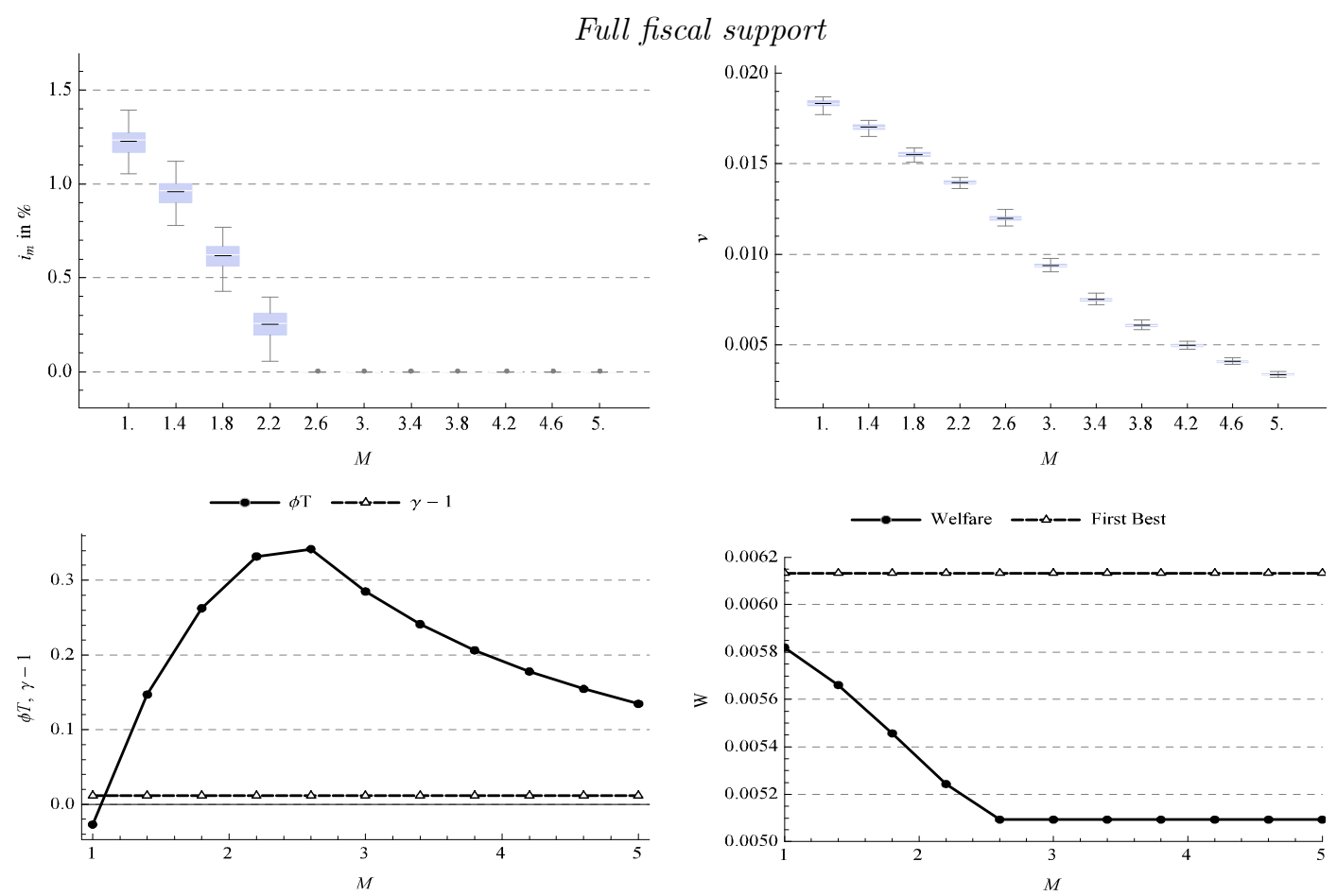

No fiscal support
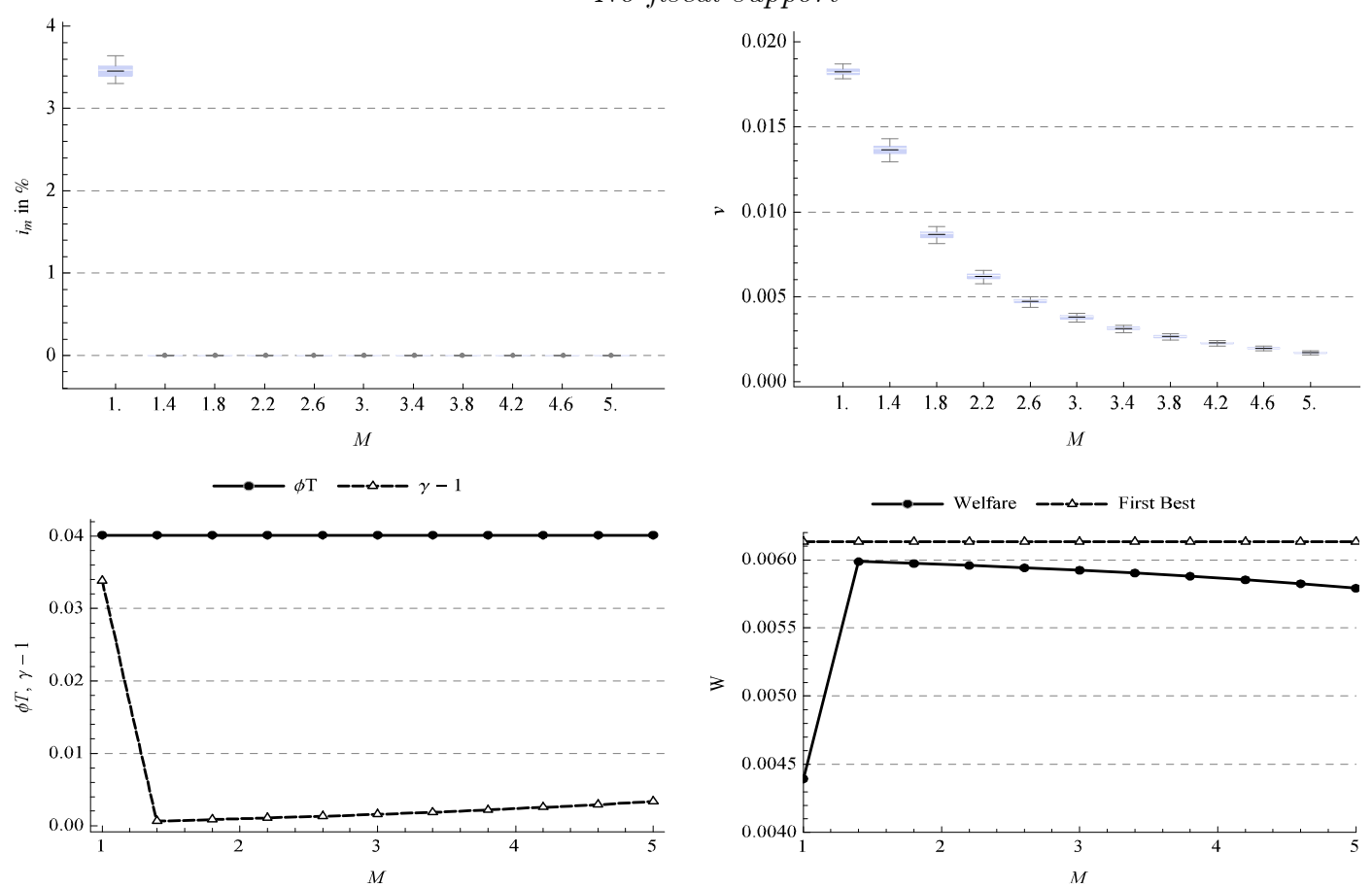

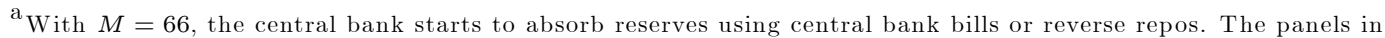
the first (third) row show the impact on the money market rate $i_{m}$ and the turnover-to-reserves ratio $v$ under full fiscal support (no fiscal support). The panels in the second (fourth) row show the impact on inflation $\gamma-1$, real transfer $\phi T$, and welfare $W$ under full fiscal support (no fiscal support). 


\section{Pre-crisis interest rate}

After we have investigated the various properties of the exit strategies, we now present our main experiment. We study the implications of returning to the pre-crisis interest rate for all strategies under full fiscal support and no fiscal support. It is assumed that the central bank has an interest rate target of $0.63 \%$, which is the average interest rate in the baseline calibration period (see Table 1). Table 3 displays our findings for all exit strategies. The first column displays the standard deviation of $i_{m}$. The second, third, and fourth columns show the turnover-to-reserves ratio $v$, the quantity of reserves $M$ at which the money market rate reaches the targeted $i_{m}$, and the bonds-to-reserves ratio $\mathcal{B}$, respectively. The fifth and sixth columns show the transfer and inflation needed to satisfy the consolidated government budget constraint (19). The seventh column reports the welfare level attained. Finally, the last column reports the yearly interest payment of the central bank to the FIs. They are calculated as follows: The money market interest rate $\left(i_{m}=0.63 \%\right)$ is multiplied by the stock of reserves that needs to be remunerated to attain the targeted interest rate. ${ }^{20}$ Except for the interest rate payments, the results for term deposits also apply for selling assets. ${ }^{21}$

TABLE 3: TARGET $i_{m}=0.63 \%^{\mathrm{a}}$

\begin{tabular}{lcccccccc}
\hline \hline Full fiscal supp. & STD $i_{m}$ & $100^{*} v$ & $M$ & $100^{*} \mathcal{B}$ & $\phi T$ & $\gamma$ & $100^{*} W$ & Payment \\
\hline IOR $\left(i_{d}=0.63 \%\right)$ & $0.00 \%$ & 0.015 & 66 & 0.060 & 0.01878 & 1.173 & 0.564 & $2.331 \mathrm{bn}$ \\
Term deposits & $0.08 \%$ & 1.544 & 1 & 3.934 & 0.26266 & 1.173 & 0.546 & $2.296 \mathrm{bn}$ \\
Cb-bills/rev.repo & $0.09 \%$ & 1.546 & 1.8 & 3.934 & 0.26266 & 1.173 & 0.546 & $2.267 \mathrm{bn}$ \\
\hline \hline No fiscal supp. & $\mathrm{STD} i_{m}$ & $100^{*} v$ & $M$ & $100^{*} \mathcal{B}$ & $\phi T$ & $\gamma$ & $100^{*} W$ & Payments \\
\hline IOR $\left(i_{d}=0.63 \%\right)$ & $0.00 \%$ & 0.024 & 66 & 0.060 & 0.04016 & 1.813 & 0.509 & $2.331 \mathrm{bn}$ \\
Term deposits & $0.06 \%$ & 1.787 & 0.6 & 6.557 & 0.04016 & 0.677 & 0.591 & $2.308 \mathrm{bn}$ \\
Cb-bills/rev.repo & $0.10 \%$ & 1.786 & 1.08 & 6.557 & 0.04016 & 0.677 & 0.591 & $2.293 \mathrm{bn}$ \\
\hline
\end{tabular}

${ }^{\mathrm{a}}$ Table 3 displays the simulation results of the various exit strategies when the central bank targets a money market rate of $0.63 \%$. The strategies are evaluated according to the following criteria: the ability to control the money market rate proxied by the standard deviation of $i_{m}$, the impact on the money market trading activity $v$, the real transfer $\phi T$, the inflation rate $\gamma$, welfare $W$, and the yearly interest payments to FIs which are necessary to attain the interest rate target with CHF 370 bn of reserves. Moreover, we also report $M$ and $B$ for the discussion of the results. Note that $v, B$, and $W$ are scaled for readability.

Inflation and welfare. The following key results emerge from our analysis: (i) Under full fiscal support, welfare is highest under IOR. (ii) With an interest rate target, selling assets or absorbing reserves using term deposits, central bank bills, or reverse repos generate the same real allocation and hence the same welfare level. (iii) Under no fiscal support, these policies generate the highest welfare level because they lead to a lower inflation rate than IOR.

To understand our welfare results, it is useful to note that from Equation (17), $o \equiv \rho \gamma / \beta$ measures FIs' opportunity cost of holding reserves. A policy that lowers $o$ improves the allocation and increases welfare. Since in equilibrium $\rho=\rho_{m}$, the interest rate target of $0.63 \%$ pins down $\rho$, and so only $\gamma$ affects this cost. Under full fiscal support, the central bank has an inflation target, and hence the opportunity costs of holding reserves are equal for all strategies. Consequently, term deposits, central bank bills and reverse repos attain the same welfare level and the same real allocation, because the bonds-to-reserves ratio $\mathcal{B}$ is the same. Furthermore, IOR generates the highest welfare level, because $\mathcal{B}$ is smaller than for the other exit strategies. Under no fiscal support and IOR, inflation and hence $o$ is higher than in all other experiments, which results in the lowest welfare level. Here, the two other strategies generate the highest welfare.

Standard deviation, turnover, and the quantity of reserves. With all strategies and in both regimes, $i_{m}$ is on target. The standard deviation is zero with IOR and strictly positive with the other two strategies. The turnover is close to zero with IOR, because the stock of reserves remains at $M=66$. In contrast, money market activity is back to the pre-QE levels when the quantity of reserves is reduced. Although turnover is not directly relevant for welfare in our model, central banks might value an active money market, since they can infer information from money market trading

\footnotetext{
${ }^{20}$ Recall that for Switzerland $M=66$, which means that the quantity of reserves is CHF $370 \mathrm{bn}$. For IOR, the total stock of reserves needs to be remunerated. Thus, the yearly interest payment to FIs is $2.331 \mathrm{bn}$. For term deposits, central bank bills and reverse repos, the amount of reserves that needs to be absorbed is only $66-M$, where $M$ is indicated in the third column of Table 3 . Due to arbitrage considerations, the interest rate paid in absorbing operations has to be equal to the money market rate.

${ }^{21}$ Our analysis only focuses on the central bank's liability side. The advantage of selling assets is that it reduces the quantity of reserves absent of interest rate payments to FIs. However, it also decreases the earnings on the asset holdings. All other exit strategies involve interest rate payments to FIs, but at the same time generate higher earnings on the asset holdings.
} 
activity that can be relevant, for example, for the calculation of transaction-based benchmark interest rates. From this point of view, IOR can be problematic.

As discussed above, when the central bank has an interest rate target, all strategies except for IOR implement the same real allocation, and hence yield the same welfare level; however, they affect the money market differently. For example, with central bank bills and reverse repos, the central bank needs to absorb less reserves than with term deposits or by selling assets, and the standard deviations of $i_{m}$ differ considerably. Finally, note that with IOR, $i_{m}$ can be maintained at the targeted level with immediate effect, while it may take considerable time to reduce the required quantity of reserves and thus to reach the interest rate target with all other strategies.

Transfers and interest payments. None of our experiments displayed in Table 3 yield a negative transfer (a tax). The highest transfers are reached under full fiscal support with selling assets or absorbing reserves using term deposits, central bank bills, or reverse repos. In all experiments, the interest payments by the central bank to FIs are of similar magnitude. Although, these lump-sum transfers and interest payments are not welfare relevant, they still might affect the public's perception of monetary policy. Large interest payments to FIs could trigger political economy reactions that could have an impact on central bank independence. For this reason, central banks should evaluate whether there are options to minimize these payments without comprising the objective to maintain the interest rate at the targeted level. For example under IOR, one could consider whether it is possible to attain the same real allocation by only remunerating a fraction of reserves. We leave the investigation of this question for future research.

\section{Conclusion}

In response to the financial crisis of 2007/08 and the subsequent sovereign debt crisis, all major central banks decreased interest rates to historically low levels and created large excess reserves. Central bankers and academics currently discuss how to raise interest rates in such an environment. The term 'exit strategy' in this context refers to the various policies that allow central banks to achieve this objective.

This paper studies exit strategies including paying IOR, selling assets purchased under QE, and absorbing reserves using term deposits, central bank bills, or reverse repos in a dynamic general equilibrium model where financial intermediaries face idiosyncratic liquidity shocks, trade reserves in the secured money market, and have access to the central bank's deposit and lending facilities. We calibrate the model to the Swiss franc repo market and perform various experiments: In one set of experiments, we absorb reserves to the level observed prior to the financial crisis. In another set of experiments, we increase the short-term interest rate to the average value that existed prior to the financial crisis. The exit strategies are evaluated with respect to (i) their welfare implications; (ii) the central bank's ability to control the money market interest rate and their impact on the money market trading activity; (iii) inflation; and (iv) taxes and interest payments to financial intermediaries. Furthermore, each strategy is investigated under two polar coordination regimes: Full fiscal support and no fiscal support.

Our main finding is that paying IOR is optimal in terms of welfare if the central bank has full fiscal support. If the central bank has no fiscal support, reducing reserves is optimal. This can be achieved by reserve-absorbing operations which hold the size of the balance sheet constant, or by selling assets which reduces the size of the balance sheet. Our findings suggest that optimality can be achieved under both regimes, irrespective of the balance sheet size.

Further findings are as follows: (i) Irrespective of the coordination regime, all exit strategies allow central banks to achieve an interest rate target, but with different levels of interest rate volatilities. (ii) In both regimes, money market trading activity under IOR is almost inexistent, while trading activity re-emerges for all other policies and for both regimes. This finding is relevant for the current discussions on benchmark interest rate reforms. To reduce the potential for manipulation and to reflect the financing conditions in an underlying market, regulators currently discuss whether references rates should be based on concluded transactions, only (see Financial Stability Board, 2013 and European Central Bank, 2013). If transaction-based benchmark interest rates are important, then, central bank bills or reverse repos would become the preferred exit strategy. ${ }^{22}$ (iii) We find that IOR under no fiscal support generates substantially more inflation than the other policies. (iv) None of our experiments required a negative real transfer (a tax) to satisfy the consolidated government budget constraint. Nevertheless, all exit strategies, except for selling assets, involve substantial interest payments from the central bank to (a few) financial intermediaries. These factors do not directly enter our welfare measure, but they are relevant for central banking practices.

\footnotetext{
${ }^{22}$ The importance of establishing credible and transaction-based benchmark interest rates has increased, as the UK Financial Conduct Authority recently announced that after 2021 it will no longer persuade, or compel, banks to submit to Libor. As a consequence, it cannot be ruled out that the most widely use benchmark interest rate worldwide will cease to exist (see UK Financial Conduct Authority, 2017).
} 
The findings of this paper contribute to the current discussion on the new regime for monetary policy implementation. In particular, it provides guidance whether monetary policy should be implemented in an excess reserve environment with IOR going forward or whether central banks should return to the pre-crisis monetary policy implementation by absorbing reserves and/or reducing the balance sheet size. 


\section{References}

[1] Afonso, G., Lagos, R., 2015. Trade Dynamics in the Market for Federal Funds. Econometrica 83 (1), 263-313.

[2] Armenter, R., Lester, B., 2017. Excess Reserves and Monetary Policy Implementation. Review of Economic Dynamics 23 (January).

[3] Armantier, O., Copeland, A., 2012. Assessing the Quality of 'Furfine-based' Algorithms. Federal Reserve Bank of New York Staff Reports 575.

[4] Bech, M., Klee, E., 2011. The Mechanics of a Graceful Exit: Interest on Reserves and Segmentation in the Federal Funds Market. Journal of Monetary Economics 58 (5), 415-431.

[5] Bech, M., Monnet, C., 2016. A Search-Based Model of the Interbank Money Market and Monetary Policy Implementation. Journal of Economic Theory 164 (C), 32-67.

[6] Benford, J., Berry, S., Nikolov, K., Young, C., 2009. Quantitative Easing. Bank of England Quarterly Bulletin 2, 90-100.

[7] Berentsen, A., Camera, G., Waller, C., 2007. Money, Credit and Banking. Journal of Economic Theory 135 (1), 171-195.

[8] Berentsen, A., Monnet, C., 2008. Monetary Policy in a Channel System. Journal of Monetary Economics 55 (6), $1067-1080$

[9] Berentsen, A., Marchesiani, A., Waller, C., 2014a. Floor Systems for Implementing Monetary Policy: Some Unpleasant Fiscal Arithmetic. Review of Economic Dynamics 17 (3), 523-542.

[10] Berentsen, A., Marchesiani, A., Huber, S., 2014b. Degreasing the Wheels of Finance. International Economic Review 55 (3), 735-763.

[11] Del Negro, M., Sims, C.A., 2015. When does a central bank's balance sheet require fiscal support. Journal of Monetary Economics, 73 (C), 1-19.

[12] Ennis, H.M., 2017. A Simple General Equilibrium Model of Large Excess Reserves. Manuscript.

[13] European Central Bank, 2013. Reference Interest Rates: Role, Challenges and Outlook. ECB Monthly Bulletin, 69-84.

[14] FOMC, 2014. Policy Normalization Principles and Plans.

[15] FOMC, 2016. Minutes of the Federal Open Market Committee (November 2016).

[16] FOMC, 2017a. Addendum to the Policy Normalization Principles and Plans.

[17] FOMC, 2017b. Minutes of the Federal Open Market Committee (September 2017).

[18] Fuhrer, L.M., Guggenheim, B., Schumacher, S., 2016. The Re-Use of Collateral in the Repo Market. Journal of Money, Credit and Banking 48 (6), 1169-1193.

[19] Fuhrer, L.M., Müller, B., Steiner, L.V., 2016. The Liquidity Coverage Ratio and Security Prices. Journal of Banking \& Finance 75 (C), 292-311.

[20] Financial Stability Board, 2013. Progress Report on the Oversight and Governance Framework for Financial Benchmark Reform. Report to G20 Finance Ministers and Central Bank Governors.

[21] Furfine, C., 2000. Interbank Payments and the Daily Federal Funds Rate. Journal of Monetary Economics 46 (2), 535-553.

[22] Guggenheim, B., Kraenzlin, S., Schumacher, S., 2011. Exploring an Uncharted Market: Evidence on the Unsecured Swiss Franc Money Market. SNB Working Paper 5.

[23] Hall, R.E., Reis, R., 2015. Maintaining Central-Bank Financial Stability under New-Style Central Banking. NBER Working Papers 21173. 
[24] ICMA, 2014. European Repo Market Survey (June 2014).

[25] Jackson, C., Sim, M., 2013. Recent Developments in the Sterling Overnight Money Market. Bank of England Quarterly Bulletin 3, 223-232.

[26] Kraenzlin, S., Nellen, T., 2010. Daytime is Money. Journal of Money, Credit and Banking 42 (8), 1689-1702.

[27] Kraenzlin, S., Nellen, T., 2015. Access Policy and Money Market Segmentation. Journal of Monetary Economics $71(\mathrm{C}), 1-12$.

[28] Kraenzlin, S., Schlegel, M., 2012. Demand for Reserves and the Central Bank's Management of Interest Rates. Swiss Journal of Economics and Statistics 148 (IV), 531-555.

[29] Lagos, R., Wright, R., 2005. A Unified Framework for Monetary Theory and Policy Analysis. Journal of Political Economy 113 (3), 463-484.

[30] Leeper, E.M., Leith, C., 2016. Understanding Inflation as a Joint Monetary and Fiscal Phenomenon. In: Taylor, J.B., Uhlig, H., (Eds.), Handbook of Macroeconomics. Elsevier, Amsterdam, pp. 2305-2415.

[31] Lester, B., Postlewaite, A., Wright, R., 2012. Information, Liquidity, Asset Prices, and Monetary Policy. Review of Economic Studies 79 (3), 1208-1238.

[32] Rocheteau, G., Wright, R., Xiao, S.X., 2017. Open Market Operations. Manuscript.

[33] Reis, R., 2016. Can the Central Bank Alleviate Fiscal Burdens? NBER Working Papers 23014.

[34] Reis, R., 2017. Funding Quantitative Easing to Target Inflation. Proceedings - Economic Policy Symposium Jackson Hole, 423-478.

[35] Sargent, T., Wallace, N., 1981. Some Unpleasant Monetarist Arithmetic. FRB of Minneapolis Quarterly Review, $531,1-17$.

[36] Thornton, D.L., 2006. When did the FOMC begin targeting the federal funds rate? What the verbatim transcripts tell us. Journal of Money, Credit 83 Banking 38 (8), 2039-71.

[37] UK Financial Conduct Authority, 2017. The Future of Libor.

[38] Williamson, S., 2016. Interest on Reserves, Interbank Lending, and Monetary Policy. Society for Economic Dynamics 2016 Meeting Papers 428. 


\section{Web Appendix A: Proofs}

In this Web Appendix, the proof of Lemma 1 and the proof of Proposition 2 is provided.

Proof of Lemma 1. For unconstrained FIs, the quantities $q_{\varepsilon}$ are derived from the first-order condition (10) by setting $\lambda_{z}=0$. Since $q_{\varepsilon}$ is increasing in $\varepsilon$, there exists a critical value $\varepsilon_{2}$ such that the FI is just constrained. Since in this case, (10) holds as well, we have $q_{\varepsilon}=\varepsilon \rho_{m} / \rho_{d}$ for $\varepsilon \leq \varepsilon_{2}$.

Next, the cut-off value $\varepsilon_{1}$ is derived. From (8) and (10), the consumption level of an FI that is unconstrained satisfies

$$
q_{\varepsilon}=\frac{\varepsilon \rho_{m}}{\rho_{d}} .
$$

The consumption level of an FI, who neither deposits nor borrows is

$$
q_{0}=\frac{m}{p} .
$$

Since (20) is increasing in $\varepsilon$, there exists an $\varepsilon_{1}$ such that

$$
\varepsilon_{1}=\frac{\rho_{d}}{\rho_{m}} \frac{m}{p} .
$$

At $\varepsilon=\varepsilon_{1}$, the FI is indifferent between depositing or borrowing. The quantity consumed by such an FI is $q_{\varepsilon_{1}}=\frac{\varepsilon_{1} \rho_{m}}{\rho_{d}}=\frac{m}{p}$.

We now calculate $\varepsilon_{2}$. At $\varepsilon=\varepsilon_{2}$, the collateral constraint is just binding. In this case, we have the following equilibrium conditions: $q_{\varepsilon_{2}}=\varepsilon_{2} \rho_{m} / \rho_{d}$ and $p q_{\varepsilon_{2}}=m+\rho_{m} \theta b$. Eliminating $q_{\varepsilon_{2}}$ yields

$$
\varepsilon_{2}=\varepsilon_{1}\left(1+\rho_{m} \frac{\theta b}{m}\right) .
$$

It is then evident that

$$
0 \leq \varepsilon_{1} \leq \varepsilon_{2} .
$$

Finally, for $\varepsilon<\varepsilon_{2}$, the quantities deposited and borrowed are derived from the budget constraint $p q_{\varepsilon}=m+z_{\varepsilon}$. Using (20) yields:

$$
z_{\varepsilon}=p\left(\rho_{m} / \rho_{d}\right)\left(\varepsilon-\varepsilon_{1}\right) .
$$

For $\varepsilon \geq \varepsilon_{2}$, we have $z_{\varepsilon}=\rho_{m} b$.

Proof of Proposition 2. The proof involves deriving equations (16) to (18). Equation (18) is derived in the proof of Lemma 1. To derive equation (16), differentiate $V_{M}(m, b)$ with respect to $m$ to obtain

$$
V_{M}^{m}(m, b)=\int_{0}^{\infty}\left[\beta V_{S}^{m}\left(m+z_{\varepsilon}+\ell_{\varepsilon}-p q_{\varepsilon}-d_{\varepsilon}, b, \ell_{\varepsilon}, d_{\varepsilon}, z_{\varepsilon} \mid \varepsilon\right)+\beta \phi^{+} \lambda_{\varepsilon}\right] d F(\varepsilon) .
$$

Then, use (7) to replace $V_{S}^{m}$ and (10) to replace $\beta \phi^{+} \lambda_{\varepsilon}$ to obtain

$$
V_{M}^{m}(m, b)=\int_{0}^{\infty} \frac{\varepsilon u^{\prime}\left(q_{\varepsilon}\right)}{p} d F(\varepsilon) .
$$

Use the first-order condition (8) to replace $p$ to obtain

$$
V_{M}^{m}(m, b)=\left(\beta \phi^{+} / \rho_{d}\right) \int_{0}^{\infty} \varepsilon u^{\prime}\left(q_{\varepsilon}\right) d F(\varepsilon) .
$$

Use (5) to replace $V_{M}^{m}(m, b)$ and replace $\phi / \phi^{+}$by $\gamma$ to obtain

$$
\frac{\rho_{d} \gamma}{\beta}=\int_{0}^{\infty} \varepsilon u^{\prime}\left(q_{\varepsilon}\right) d F(\varepsilon)
$$


Finally, note that $u^{\prime}(q)=1 / q$ and replace the quantities $q_{\varepsilon}$ using Lemma 1 to obtain (16), which is replicated here:

$$
\frac{\rho_{d} \gamma}{\beta}=\int_{0}^{\varepsilon_{2}} \frac{\rho_{d}}{\rho_{m}} d F(\varepsilon)+\int_{\varepsilon_{2}}^{\infty} \frac{\varepsilon}{\varepsilon_{2}} \frac{\rho_{d}}{\rho_{m}} d F(\varepsilon) .
$$

To derive (17), note that in any equilibrium with a strictly positive demand for reserves and bonds, we must have $\rho V_{M}^{m}(m, b)=V_{M}^{b}(m, b)$. This arbitrage equation is used to derive (17). $V_{M}^{m}(m, b)$ was already derived in (23). To get $V_{M}^{b}(m, b)$ differentiate $V_{M}(m, b)$ with respect to $b$ to obtain

$$
V_{M}^{b}(m, b)=\int_{0}^{\infty}\left[\beta V_{S}^{b}\left(m+\ell_{\varepsilon}-p q_{\varepsilon}-d_{\varepsilon}, b, \ell_{\varepsilon}, d_{\varepsilon} \mid \varepsilon\right)+\rho_{m} \beta \phi^{+} \lambda_{z}\right] d F(\varepsilon) .
$$

Use (7) to replace $V_{S}^{b}$ to obtain

$$
V_{M}^{b}(m, b)=\beta \phi^{+} \int_{0}^{\infty}\left(1+\rho_{m} \lambda_{z}\right) d F(\varepsilon)
$$

Then, use (10) to replace $\lambda_{z}$, and rearrange to obtain

$$
V_{M}^{b}(m, b)=\int_{0}^{\varepsilon_{2}} \beta \phi^{+} d F(\varepsilon)+\beta \phi^{+} \int_{\varepsilon_{2}}^{\infty}\left(\rho_{m} / \rho_{d}\right) \varepsilon u^{\prime}\left(q_{\varepsilon}\right) d F(\varepsilon) .
$$

Equate $\rho V_{M}^{m}(m, b)=V_{M}^{b}(m, b)$ and simplify to obtain

$$
\rho \int_{0}^{\infty} \varepsilon u^{\prime}\left(q_{\varepsilon}\right) d F(\varepsilon)=\int_{0}^{\varepsilon_{2}} \rho_{d} d F(\varepsilon)+\int_{\varepsilon_{2}}^{\infty} \rho_{m} \varepsilon u^{\prime}\left(q_{\varepsilon}\right) d F(\varepsilon) .
$$

Note that $\int_{0}^{\infty} \varepsilon u^{\prime}\left(q_{\varepsilon}\right) d F(\varepsilon)=\rho_{d} \gamma / \beta$ and rearrange to obtain

$$
\frac{\rho \gamma}{\beta}=\int_{0}^{\varepsilon_{2}} d F(\varepsilon)+\int_{\varepsilon_{2}}^{\infty}\left(\rho_{m} / \rho_{d}\right) \varepsilon u^{\prime}\left(q_{\varepsilon}\right) d F(\varepsilon) .
$$

Finally, use Lemma 1 to obtain (17), which is replicated here:

$$
\frac{\rho \gamma}{\beta}=\int_{0}^{\varepsilon_{2}} d F(\varepsilon)+\int_{\varepsilon_{2}}^{\infty}\left(\varepsilon / \varepsilon_{2}\right) d F(\varepsilon) .
$$

\section{Web Appendix B: Consolidated government budget constraint and welfare}

In this Web Appendix, we show how to calculate the real transfers in a steady state, and we derive an expression for welfare. Furthermore, we describe in more detail the difference between full fiscal support and no fiscal support.

Consolidated government budget constraint in the steady state. Here, we show how to derive (19) from the consolidated government budget constraint (3) which we replicate here:

$$
\phi T=\phi M^{+}-\phi M+\phi \rho B^{+}-\phi B-\left(1 / \rho_{d}-1\right) \phi D+\left(1 / \rho_{\ell}-1\right) \phi L .
$$

In a first step, note that in any equilibrium $D=M+L$. This allows us to write (3) as follows: 


$$
\phi T=\phi M^{+}-\phi M / \rho_{d}+\phi \rho B^{+}-\phi B+\left(1 / \rho_{\ell}-1 / \rho_{d}\right) \phi L .
$$

In a steady state, $\phi M^{+}=\phi M$ and $B^{+} / M^{+}=B / M=\mathcal{B}$. Accordingly, we have

$$
\phi T=\phi M\left[\gamma-1 / \rho_{d}+(B / M)(\rho \gamma-1)+\left(1 / \rho_{\ell}-1 / \rho_{d}\right) L / M\right]
$$

where $\phi M=\frac{\rho \varepsilon_{1}}{\beta}$. To see this, recall that $M$ is the stock of reserves at the beginning of the current-period settlement market, and $\phi$ is the price of reserves in terms of the generic good that is traded in this market. From Lemma 1, we have $\varepsilon_{1}=\frac{\rho_{d}}{\rho_{m}} \frac{m}{p}$, where $m=M^{+}$, since the quantity of reserves in the DM of period $t$ is $M^{+}$. Furthermore, from (8), sellers are indifferent as to how much they sell in the goods market if $p \beta \phi^{+} / \rho_{d}=1$, where $\phi^{+}$is the price of reserves in the next-period settlement market. By combining these two equations, we obtain $\varepsilon_{1}=\frac{\beta \phi^{+} M^{+}}{\rho_{m}}$. Finally, in a steady state $\rho=\rho_{m}$ and $\phi M=\phi^{+} M^{+}$, and so $\phi M=\frac{\rho \varepsilon_{1}}{\beta}$. Use this expression to rewrite (25) to obtain (19) which we replicate here for ease of reference:

$$
\phi T=\frac{\rho \varepsilon_{1}}{\beta}\left[\gamma-1 / \rho_{d}+(B / M)(\rho \gamma-1)+\left(1 / \rho_{\ell}-1 / \rho_{d}\right) L / M\right]
$$

How we use this expression depends on whether we study full fiscal support or no fiscal support.

Full fiscal support versus no fiscal support. Under full fiscal support, we only use (26) to calculate the calibrated value of $\phi T$ which we denote $\overline{\phi T}$. To derive this value, note that in the steady state of the baseline calibration $\left(1 / \rho_{\ell}-1 / \rho_{d}\right) L / M=0$, since $L=0$. When we report how a change in policy affects $\phi T$, we recalculate the endogenous variables $\rho$ and $\varepsilon_{1}$ from Proposition (2). For example, if we decrease $\rho_{d}$, we take into account the direct effect and the indirect effect via $\rho$ and $\varepsilon_{1}$ in (26).

Under no fiscal support, we assume that the real transfer is kept constant at the calibrated value $\overline{\phi T}$. Furthermore, in response to any monetary policy change, we assume that the inflation rate adjusts endogenously. To capture this assumption, we solve $(26)$ for $\gamma(\overline{\phi T})$ as follows

$$
\gamma(\overline{\phi T})=\frac{\frac{\overline{\phi T} \beta}{\rho \varepsilon_{1}}+1 / \rho_{d}+(B / M)-\left(1 / \rho_{\ell}-1 / \rho_{d}\right) L / M}{1+(B / M) \rho} .
$$

We then use $\gamma(\overline{\phi T})$ to substitute $\gamma$ in Proposition (2). This allows us to calculate how all endogenous variables change in response to a policy change under the assumption that the real transfer $\phi T$ is constant.

Welfare. Here it is shown that welfare has the same functional form under full and no fiscal support. Welfare, measured at the beginning of the settlement market, satisfies

$$
(1-\beta) W=\int\left[\varepsilon\left(q_{\varepsilon}\right)-q_{\varepsilon}+\left(x_{\varepsilon}-h_{\varepsilon}\right)\right] d F(\varepsilon) .
$$

To derive welfare, the integral $\int\left(x_{\varepsilon}-h_{\varepsilon}\right) d F(\varepsilon)$ needs to be identified. In the settlement market, FIs solve the following optimization problem:

$$
\begin{gathered}
V_{S}(m, b, \ell, d, z)=\max _{h, x, m \prime, b l} x-h+V_{M}\left(m^{\prime}, b^{\prime}\right) \\
\text { s.t. } x+\phi m^{\prime}+\phi \rho b^{\prime}=h+\phi m+\phi b+\phi d / \rho_{d}-\phi \ell / \rho_{\ell}-\phi z / \rho_{m}+\phi \tau M .
\end{gathered}
$$

Accordingly, $\int\left(x_{\varepsilon}-h_{\varepsilon}\right) d F(\varepsilon)$ satisfies

$$
\int\left(x_{\varepsilon}-h_{\varepsilon}\right) d F(\varepsilon)=\int\left(\phi m_{\varepsilon}-\phi m^{\prime}+\phi b-\phi \rho b^{\prime}+\phi d_{\varepsilon} / \rho_{d}-\phi \ell_{\varepsilon} / \rho_{\ell}-\phi z_{\varepsilon} / \rho_{m}+\phi \tau M\right) d F(\varepsilon) .
$$

Market clearing in the money market implies that $\int\left(\phi z_{\varepsilon} / \rho_{m}\right) d F(\varepsilon)=0$. Note that FIs hold no reserves when they enter the settlement market, since all reserves are deposited at the deposit facility. Accordingly, $m_{\varepsilon}=0$ and $d_{\varepsilon}=\tilde{m}_{\varepsilon}+\ell_{\varepsilon}$, 
where $\tilde{m}_{\varepsilon}$ are reserves deposited at the deposit facility that are not borrowed from the central bank. Accordingly,

$$
\int\left(x_{\varepsilon}-h_{\varepsilon}\right) d F(\varepsilon)=\int\left(-\phi m^{\prime}+\phi b-\phi \rho b^{\prime}+\phi\left(\tilde{m}_{\varepsilon}+\ell_{\varepsilon}\right) / \rho_{d}-\phi \ell_{\varepsilon} / \rho_{\ell}+\phi \tau M\right) d F(\varepsilon) .
$$

In a steady state, $m^{\prime}=M^{+}, b^{\prime}=B^{+}, b=B$, and $\int \tilde{m}_{\varepsilon} d F(\varepsilon)=M, \int \ell_{\varepsilon} d F(\varepsilon)=L$. Accordingly, we get

$$
\int\left(x_{\varepsilon}-h_{\varepsilon}\right) d F(\varepsilon)=\phi M / \rho_{d}-\phi M^{+}+\phi B-\phi \rho B^{+}-\left(1 / \rho_{\ell}-1 / \rho_{d}\right) \phi L+\phi \tau M .
$$

Consider, first, the case of full fiscal support. From the consolidated government budget constraint, we have

$$
\tau M=M^{+}-M / \rho_{d}+\rho B_{g}^{+}-B_{g}+\rho B_{c}^{+}-B_{c}+\left(1 / \rho_{\ell}-1 / \rho_{d}\right) \phi L .
$$

This implies that $\int\left(x_{\varepsilon}-h_{\varepsilon}\right) d F(\varepsilon)=0$, since $B=B_{c}+B_{g}$. Accordingly, this yields

$$
(1-\beta) W=\int\left[\varepsilon\left(q_{\varepsilon}\right)-q_{\varepsilon}\right] d F(\varepsilon) .
$$

Consider, next, the case of no fiscal support. From the consolidated government budget constraint, we have

$$
\tau \phi M=\overline{\phi T}
$$

where $\phi T$ is a constant. That is,

$$
\int\left(x_{\varepsilon}-h_{\varepsilon}\right) d F(\varepsilon)=\phi M / \rho_{d}-\phi M^{+}+\phi B-\phi \rho B^{+}-\left(1 / \rho_{\ell}-1 / \rho_{d}\right) \phi L+\overline{\phi T} .
$$

From the consolidated government budget constraint, we have

$$
\phi T=\phi M^{+}-\phi M / \rho_{d}+\phi \rho B^{+}-\phi B+\left(1 / \rho_{\ell}-1 / \rho_{d}\right) \phi L .
$$

Furthermore, no fiscal support requires that in any period $\phi T=\overline{\phi T}$. This implies that $\int\left(x_{\varepsilon}-h_{\varepsilon}\right) d F(\varepsilon)=0$, since $B=B_{c}+B_{g}$. Accordingly, this yields

$$
(1-\beta) W=\int\left[\varepsilon\left(q_{\varepsilon}\right)-q_{\varepsilon}\right] d F(\varepsilon) .
$$

\begin{tabular}{|c|c|c|}
\hline Description & Period & Frequency \\
\hline SARON & Jan 2005 - Dec 2013 & Daily \\
\hline Overnight SNB Special Rate & Jan 2005 - Dec 2013 & Daily \\
\hline Inflation (year-on-year change) & Jan 2005 - Dec 2013 & Monthly \\
\hline Real interest rate & Q3 2005 - Q4 2013 & Quarterly \\
\hline Money market transaction data & Jan 2005 - Dec 2013 & Daily \\
\hline Central bank reserves & Jan 2005 - Dec 2013 & Daily \\
\hline
\end{tabular}

This clearly shows that the coordination regime choice does not affect the functional form of the welfare function.

\section{Web Appendix C: Data}

The model is adapted to replicate the elementary features of the Swiss franc repo market and monetary policy implementation by the SNB. The data used for the calibration are described in Table C.1 and are provided by Eurex Ltd., the Swiss Federal Statistical Office (SFSO), the SNB and SIX Ltd.

TABle C.1: Data ${ }^{\mathrm{a}}$ 


\section{Web Appendix D: Calibration and simulation strategy}

In this Web Appendix, we describe in more detail the calibration and simulation strategy.

To assess the model's in-sample fit, a finite number $n^{t}$ liquidity shocks is drawn from a log-normal distribution with the calibrated moments $\mu$ and $\sigma$. The liquidity shocks are assumed to be unexpected and we are abstracting from aggregate shocks. Let $\Omega^{t}$ denote the set of liquidity shocks $\varepsilon$ drawn in period $t$. For each $\varepsilon \in \Omega^{t}$, Lemma 1 is used to calculate the net borrowing $z_{\varepsilon}$. Given the various $z_{\varepsilon}$, the market clearing condition (14) is used to calculate the money market rate $i_{m}^{t}$. Since each individual trade that occurs under $\Omega^{t}$ is known, we can also calculate the turnover-to-reserves ratio $v^{t}$ from (12) that occurs in period $t$.

The simulated turnover-to-reserves is derived as follows: A finite number $n_{t}=4,000$ of liquidity shocks $\varepsilon$ is drawn from the calibrated $\log$-normal distribution, and Lemma 1 is used to calculate $z_{\varepsilon} / M$ for each $\varepsilon$. Then, we calculate the sum over all strictly positive $z_{\varepsilon} / M$ and divide the resulting sum by $n_{t}$. Finally, we repeat this process $T$ times. To map the data to the model, the empirical turnover-to-reserves ratio is calculated as follows. The overnight turnover is divided by the number of active FIs per day. Subsequently, the daily average turnover per active FI is normalized by the stock of reserves. Finally, note that we can also calculate the volumes lent or borrowed as follows: A set of liquidity shocks $\varepsilon$ with $n_{t}=4,000$ is drawn from the calibrated log-normal distribution. Then, Lemma 1 is used to calculate $z_{\varepsilon} / M$ for each $\varepsilon$.

To generate a sequence of $i_{m}^{t}$ and $v^{t}$, the sampling exercise is repeated for $T$ periods. We report the means and the standard deviations of $i_{m}^{t}$ and $v^{t}$ and compare them with the empirical counterparts of the baseline sample. For the calibration, we assume that all liquidity shocks from the underlying distribution are present. In contrast, when we simulate the model, we draw finite sets of liquidity shocks $\Omega^{t}, t=1, . ., T$, from the underlying distribution. This leads to variability in the money market rate and the turnover-to-reserves ratio across periods. We have chosen this calibration strategy, because it is easy to implement and because there is a long tradition in macroeconomics of calibrating a dynamic stochastic general equilibrium model to the non-stochastic steady state.

The choice of the sample size $n^{t}$ affects the standard deviation of $i_{m}$ and $v$. In particular, the standard deviation converges to zero as the sample size is increased to infinity. To pin down $n^{t}$, we choose $n^{t}=4,000$ such that the standard deviation of $i_{m}$ matches the empirical standard deviation of $i_{m}^{e}$. The number $T$ is chosen to fit the number of trading days in the baseline sample. Note that in the model, $n^{t}$ represents the number of active FIs in the money market at time $t$. This number is much higher than the 32 FIs that were active on an average day. Potential reasons why $n^{t}$ has to be set higher to match the empirical standard deviation of $i_{m}^{e}$ are the SNB's fine-tuning operations. Fine-tuning operations were conducted when the money market rate deviated too far from the targeted level and thus limits the standard deviation of the money market rate.

\section{Web Appendix E: Comparative statics}

Based on the calibrated parameters, we can explore graphically how the demand and the supply of reserves react to exogenous shocks to $M, \mu, B$, and $\sigma$. For each figure shown below, the money market rate $i_{m}$ is displayed on the horizontal axis and the turnover-to-reserves ratio $v$ is displayed on the vertical axis. Demand and supply are shown for the calibrated parameters (solid lines) and for the variation in the parameter under consideration (dashed lines). In drawing these figures, we keep the value of reserves $\phi$ constant.

The panel on the left-hand side of Figure E.1 displays the effect of a reduction in $M$ of one percent. In this case, the demand for reserves increases (the blue curve shifts upwards) and the supply decreases (the red curve shifts downwards). As a result, $i_{m}$ unambiguously increases. The effect on $v$ is ambiguous, but in the present case, the numerical comparison suggests a slight decrease in $v$.

The effect of an increase in $\mu$ is very similar and is shown in the panel on the right-hand side of Figure E.1. If the average liquidity shock increases, the demand for reserves increases and the supply of reserves decreases. Consequently, $i_{m}$ unambiguously increases. The effect on $v$ is ambiguous, but in the present case a decrease in $v$ is found. 
Figure E.1: Comparative statics (I)
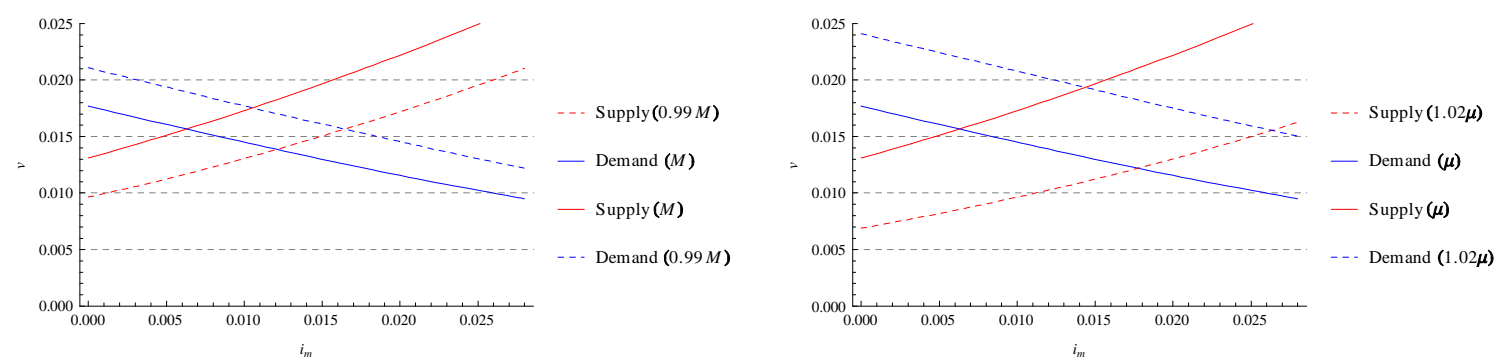

${ }^{\mathrm{a}}$ Based on the calibrated parameters, the impact of a change in $M$ (left-hand-side panel) and a change of the average liquidity shock $\mu$ (right-hand-side panel) on the demand and the supply of reserves in the money market is displayed.

The panel on the left-hand side of Figure E.2 displays the effect of doubling $B$. A change in $B$ has no effect on the supply curve. It only increases the demand for reserves, since fewer FIs are collateral-constrained. Here, the comparative statics are unambiguous: Both $i_{m}$ and $v$ increase.

The panel on the right-hand side of Figure E.2 displays the effect of a decrease in $\sigma$ to $0.5 \sigma$. If the standard deviation of the liquidity shock decreases, the need for reallocating reserves between FIs decreases. Consequently, the demand for reserves and the supply of reserves decrease. This unambiguously decreases $v$, but the effect on $i_{m}$ is ambiguous. In the present case, an increase in $i_{m}$ is found.

Figure E.2: Comparative Statics (II) ${ }^{\mathrm{a}}$
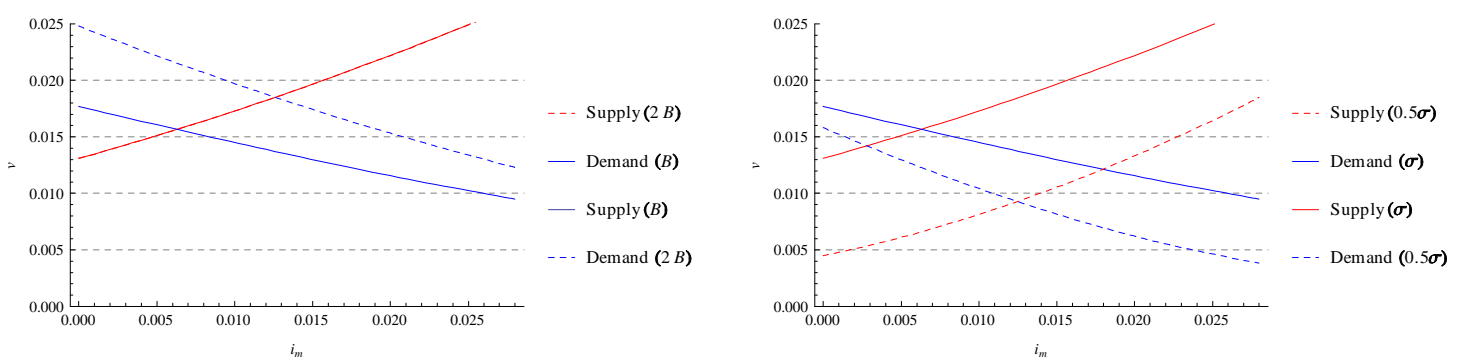

${ }^{a}$ Based on the calibrated parameters, the impact of a change in the quantity of bonds $B$ held by FIs (left-hand-side panel) and a change of the standard deviation of the liquidity shock $\sigma$ (right-hand-side panel) on the demand and the supply of reserves in the money market is displayed.

\section{Web Appendix F: The Swiss franc repo market}

The Swiss franc repo market (SFRM) is the secured money market for central bank reserves. FIs trade in this market to fulfill minimum reserve requirements and in response to liquidity shocks. Trades are concluded on an electronic trading platform with a direct link to the real-time gross settlement payment system (RGTS) called Swiss Interbank Clearing (SIC) and the central securities depository (CSD) called Swiss Security Services (SIS). Transactions concluded on the platform are settled by SIC and SIS where the latter also serves as the triparty-agent. ${ }^{23}$ On the same platform, the SNB conducts its open market operations and offers its standing facilities. The SFRM represents the relevant money market in Swiss francs in terms of volume and participation. This is especially true since the financial crisis, when the unsecured money market collapsed (see Guggenheim et al., 2011)

Domestic banks, insurance companies and federal agencies, as well as banks domiciled abroad, may access the SFRM. In 2014, 152 FIs had access of which 150 also had access to the SNB's open market operations and standing facilities (for a summary of SNB's access policy, see Kraenzlin and Nellen, 2015). Tradable maturities range from overnight to twelve months. In this paper, the focus is on the overnight maturity since approximately two-thirds of the daily turnover is overnight. ${ }^{24}$ Approximately $99 \%$ of all transactions on the platform are secured by securities that belong to a general collateral (GC) basket, the so-called 'SNB GC' basket. This is the same collateral basket that the SNB accepts in its

\footnotetext{
${ }^{23}$ The triparty-agent manages the collateral selection, the settlement, the ongoing valuation of the collateral and the initiation of margin calls.

${ }^{24}$ The overnight market is the origin of the term structure of interest rates. It is the most important interest rate for the pricing of many financial products.
} 
open market operations and standing facilities. The collateral standard within the SNB GC is homogeneous, because the SNB sets high requirements with respect to the rating and the market liquidity of eligible securities. ${ }^{25}$

The 'Swiss Average Rate Overnight' (SARON) is the money market rate for the overnight maturity, which is calculated as a volume-weighted interest rate based on overnight trades and quotes in the SFRM. The 'Overnight SNB Special Rate' is the interest rate in SNB's lending facility and is calculated based on the SARON plus 50 basis points. ${ }^{26}$

Figure F.1 displays the SARON, the Overnight SNB Special Rate, and the 20-day moving average of the overnight turnover for the period from 2005 to 2013 . For that period, the average daily overnight turnover was CHF $3.2 \mathrm{bn}$, and 30 FIs were active on an average day. In total, 107,517 overnight trades were concluded.

\section{FiguRE F.1: StYLIZED FACTS ${ }^{\mathrm{a}}$}

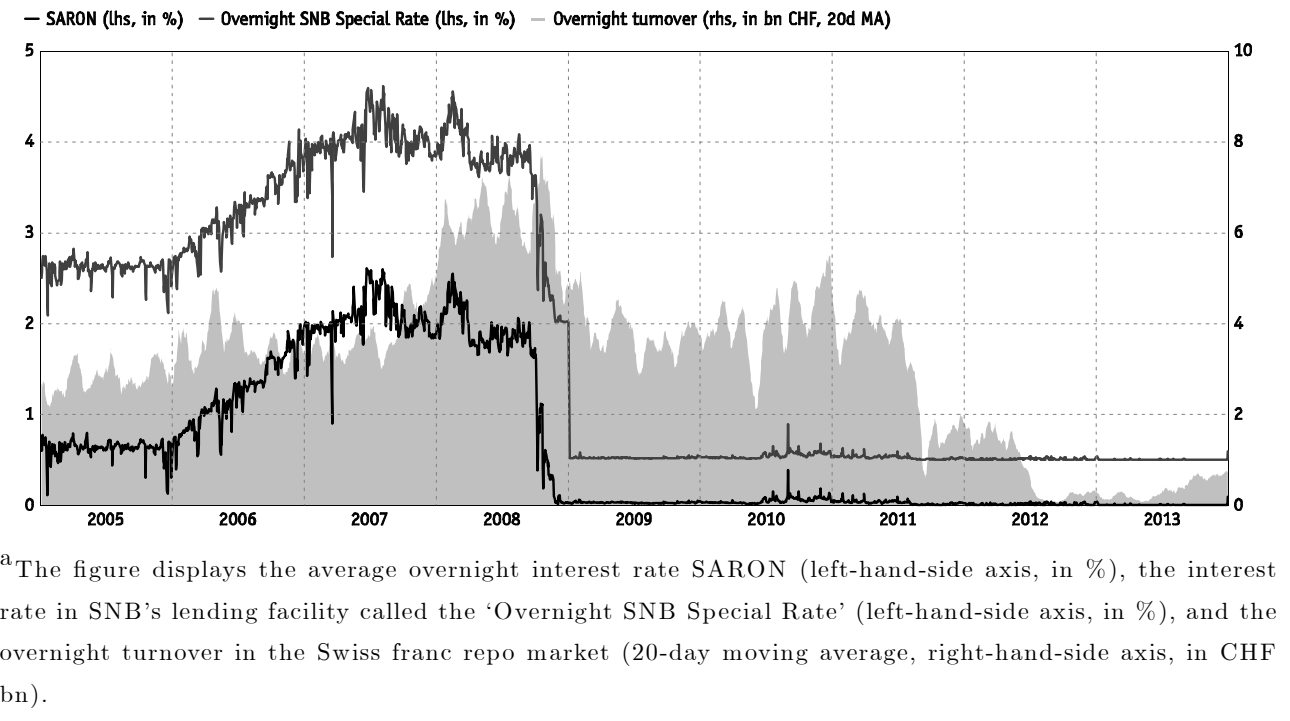

Although, the SNB's key policy rate is not the SARON, but a target range of the Swiss franc three-month Libor, the SARON reflects the SNB's monetary policy stance, since the SNB controls Libor via daily repo auctions in the SFRM. Furthermore, to keep track of prevailing monetary conditions, the SNB monitors the intraday development of the SARON and, if needed, conducts fine-tuning operations in the SFRM by placing or accepting overnight quotes.

Trading protocol. Trades in the SFRM are initiated by placing or accepting binding offers (so-called quotes) or by sending offers (so-called addressed offers, AOs) to counterparties. Quotes are entries that are placed on the electronic trading platform which indicate the maturity, the interest rate, the trade volume, the collateral basket, and the identity of the FI that has entered the quote. Quotes are collected in an order book which lists bid- and ask quotes for all maturity segments and collateral baskets. A trade upon a quote can be executed by accepting a quote via a click. ${ }^{27}$ AOs are price offers that can be sent to selected counterparties and hence are not visible for other FIs. As in the case of quotes, AOs specify the maturity, the interest rate, the trade volume, and the collateral basket. AOs can be negotiated upon by sending a counteroffer to the AO sender.

The terms-of-trades of all past trades (based on quotes and AOs) are viewable on the platform. The platform thus guarantees that all FIs have the same information set. In particular, at any time during the day, they can ascertain the maturities, interest rates, traded volumes, and collateral baskets used in all past trades. Current market conditions are likewise common knowledge thanks to the order book.

Competitive market. For several reasons, the SFRM is not an OTC market with search and bargaining frictions. First, an analysis of all overnight trades between 2005 and 2013 reveals that three-quarters of overnight trades are based on quotes, and hence, no bargaining on the terms-of-trades takes place. ${ }^{28}$ Second, in an OTC market, traders meet

\footnotetext{
${ }^{25}$ For a description of SNB eligible collateral, see Fuhrer et al. (2017).

${ }^{26}$ Until 2009, the Overnight SNB Special Rate was calculated based on the SARON plus 200 basis points.

${ }^{27}$ Theoretically, FIs can choose to reveal their quotes only to a restricted group of counterparties. However, this is very rarely done in practice.

${ }^{28} \mathrm{~A}$ comparison to longer maturities suggests that the relative number of quote based trades is largest in the overnight maturity and decreases the longer the term of the transaction. In the case of the one-week (one-month, six-month) maturity, $65 \%$ (50\%, $43 \%$ ) are based on quotes.
} 
bilaterally and the amount borrowed must be equal to the amount lent in each match. In contrast, in the SFRM, on an average day 13 borrowing and 17 lending FIs are active on the platform. This implies asymmetric trading volumes: the average borrower borrows more than the average lender lends. ${ }^{29}$ Third, deviations in the interest rates of individual overnight transactions from the SARON are very small - the average daily absolute deviation between 2005 and 2013 is $0.042 \% .{ }^{30}$ Fourth, for the same period, the average daily bid and ask volume in the order book is CHF 5.5 bn which suggests that an individual FI is not able to affect the overnight rate substantially. Fifth, the access to the platform is open to many FIs. In other words, even though on an average day only 30 banks are active, many FIs continuously monitor the market and are ready to step in if the market conditions provide attractive borrowing and lending opportunities. Sixth, all loans are secured. Consequently, counterparty risk is negligible.

In our view, the six reasons discussed above clearly indicate that the SFRM is best modeled as a competitive market, and not as an OTC market. There are no informational frictions, since all FIs have the same information on past market activities and current market conditions. Furthermore, the large number of market participants and the small price dispersion suggest that no FI has market power. FIs also tend to be indifferent to their choice of trading partners: this is explained by the high collateral standard and the absence of counterparty risk.

Mapping the SFRM to the theoretical model. Our theoretical model is motivated by the elementary features of the SFRM and the SNB's monetary policy implementation. First, at the beginning of the day all outstanding overnight loans are settled. Second, the SFRM operates between 7 am and $6 \mathrm{pm} .{ }^{31}$ Third, the SNB controls the stock of reserves by conducting open market operations, typically at $9 \mathrm{am}$, or fine-tuning operations to counter undesired fluctuations in the overnight rate on an irregular basis. Fourth, after the money market has closed, the SNB offers its lending facility for an additional 15 minutes. This is the last opportunity for FIs to acquire overnight reserves for the same business day to settle outstanding short positions in the payment system. ${ }^{32}$

The SFRM in the structural liquidity surplus environment. In response to the financial crisis of 2007/2008 and the subsequent sovereign debt crisis, the SNB increased reserves via foreign exchange purchases from roughly CHF 5.62 bn in 2005 to CHF 370 bn in 2013 (a factor of 66). As a result, the banking system holds large excess reserves and is in a so-called structural liquidity surplus, which no longer requires the SNB to conduct reserve providing operations. Money market interest rates are near zero, and money market activity collapsed as shown in Figures F.1 and F.2.

Figure F.2: SNB'S RESPONSE IN THE CRISIS ${ }^{\mathrm{a}}$

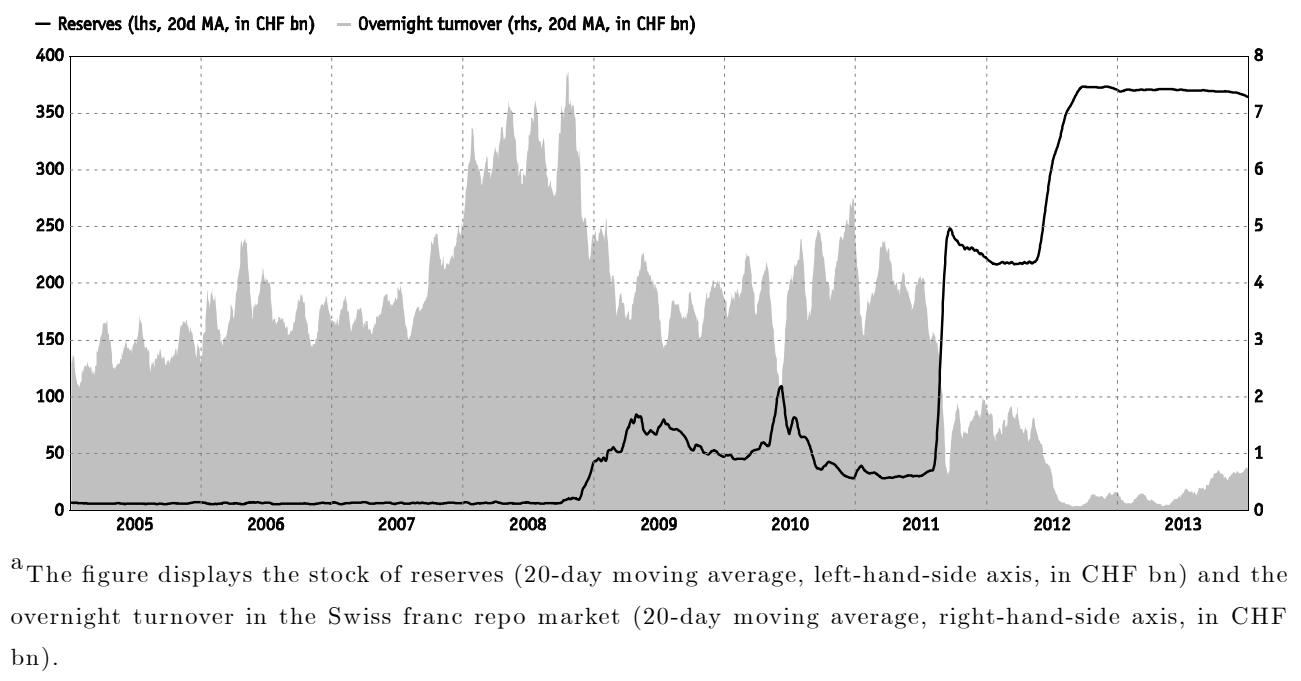

\footnotetext{
${ }^{29}$ One way to capture this stylized fact in an OTC market would be to introduce sequential matching; i.e., FIs are matched multiple times in one period.

${ }^{30}$ The comparison to other maturities shows that the deviation is smallest in the overnight maturity and increases the longer the term of the transaction. The respective figure for the one-week (one-month, six-month) maturity is $0.07 \%(0.1 \%, 0.27 \%)$.

${ }^{31}$ At 7:50 a.m. the repayment of all outstanding overnight transactions is automatically triggered. Transactions are rarely concluded between 7 am and 8 am (see Kraenzlin and Nellen, 2010).

${ }^{32}$ Short positions remaining at the end of the day must be settled the following business day and are subject to a penalty that is agreed upon bilaterally on the basis of the SARON. The stigma associated with non-settled payments imposes a further penalty which became very pronounced during the financial crisis.
} 